\title{
ON THE VISCOSITY SOLUTIONS OF HYBRID HJB EQUATIONS ARISING IN OPTIMAL CONTROL OF LOSS NETWORKS*
}

\author{
ZHONGJING MA ${ }^{\dagger}$, PETER E. CAINES ${ }^{\ddagger}$, AND ROLAND P. MALHAMÉ §
}

\begin{abstract}
Call admission and routing controls for loss (circuit-switched) networks with semiMarkovian, multi-class call arrivals and general connection durations, were formulated as optimal stochastic control problems in $[12,13]$. Each of the resulting so-called (network) hybrid HJB equations corresponds to a collection of coupled first-order partial differential equations for which, when it exists, the continuously differentiable value function is a solution to the associated hybrid HJB equations. In general, the smoothness of the value functions and uniqueness of the solutions to the hybrid HJB equations may not hold. In this paper, viscosity solutions to a general class of hybrid HJB equations are developed and under mild conditions it is shown that the value function is continuous and, further, any continuous value function is the unique viscosity solution to the hybrid HJB equations.
\end{abstract}

1. Introduction. This paper is concerned with the existence, uniqueness and smoothness properties of a class of Hamilton-Jacobi-Bellman (HJB) equations arising in the control of stochastic loss (circuit-switched) networks. In earlier work on networks, classes of call admission and routing control problems in loss networks with Poisson call request processes and exponentially distributed connection durations were treated within the framework of the the Markov decision process (MDP) methodology (see e.g. [7], [6], [9], [1] and references herein). However it is of interest to treat more general network system dynamics, for instance those for where multi-class call request processes are defined and have the nature of renewal processes, and where the connection duration processes are generally distributed. For a class of such networks the associated problems of call admission and routing control where formulated as optimal stochastic control (OSC) problems in $[12,13,16]$.

Within the network control framework of $[12,13,16]$ the network system possesses a (hybrid) state process, denoted $\mathbf{x}$, which is composed of three parts: (i) the positive integer valued part $\mathbf{n} \in \mathcal{N} \subset \mathbb{Z}_{+}^{R}$, with $\mathbb{Z}_{+} \triangleq\{0,1,2, \cdots\}$, for some positive integer $R$, which denotes the number of connections on any route of the network; (ii) the real positive valued vector $\zeta, \zeta \in \mathbb{R}_{+}^{d}$ for some positive integer $d$ dependent upon the value of $\mathbf{n}$, which comprises the durations of all active connections in the network and the elapsed times from the last call requests; and (iii) $e$, the call request and connection

\footnotetext{
${ }^{*}$ This work was supported by NSERC Discovery and Strategic Grants.

$\dagger$ Zhongjing $\mathrm{Ma}$ is with Centre for Intelligent Machines (CIM), Group for Research in Decision Analysis (GERAD) and Department of Electrical and Computer Engineering, McGill University, Montreal, Canada, E-mail: mazhj@cim.mcgill.ca

${ }^{\ddagger}$ Peter E. Caines, the corresponding author, is with CIM, GERAD and Department of Electrical and Computer Engineering, McGill University, Montreal, Canada, E-mail: peterc@cim.mcgill.ca

$\S$ Roland P. Malhamé is with GERAD and Département de génie électrique, Polytechnique, Montreal, Canada, E-mail: roland.malhame@polymtl.ca
} 
ending event process. Furthermore in case that a control law is implemented at instant $t$, the components of the state process $\mathbf{n}$ and $\zeta$ are changed to distinct valued $\widehat{\mathbf{n}}$ and $\widehat{\zeta}$ respectively following the controlled state transition equation.

Based upon the above specific characteristics, the resulting network control problems under study in $[12,13,16]$ fall within the framework of stochastic point process control [3]. As such there are natural parallels with (i) stochastic optimal control problems in the standard diffusion process case where the associated HJB equations are second order PDEs, [5, 8], and (ii) classical deterministic optimal control problems where the associated HJB equations are first order PDEs [4]. In the present context, the hybrid HJB equations which result from network OSC problems consist of a collection $V$ of coupled first-order partial differential equations. Now if the members of the corresponding collection of value functions $V=\left\{V_{\mathbf{n}} ; \mathbf{n} \in \mathcal{N}\right\}$ are continuously differentiable, i.e. $V_{\mathbf{n}} \in C^{1}\left([0, T] \times \mathbb{R}_{+}^{d(\mathbf{n})}\right)$, for all $\mathbf{n} \in \mathcal{N}$, then it is the case that $V$ is a set of classical solutions of the associated hybrid HJB equations (see [13, 14]), however, in general, the value functions are not smooth and, even if classical solutions of the hybrid HJB equations exist they may not be unique.

In this paper, we study viscosity solutions of the hybrid HJB equations; these viscosity solutions are to be distinguished from those that arise in the following settings: (i) the HJB equations for deterministic optimal control problems [4], (ii) the Hamilton-Jacobi equations analyzed in the original formulation of the viscosity solution notion by (Crandall and Lions [2]), (iii) the HJB equations of optimal stochastic control problems in the diffusion process case (see $[8,5,20])$.

More specifically, we first show that under mild conditions the collection of value functions $V$ is continuous and, second, prove that under slightly stronger conditions, a continuous value function is the unique viscosity solution to the hybrid HJB equation; the reader is referred to Figure 1.1 for the diagram of the relation of the main results of this paper.

Note that parallel to the viscosity solution analysis for the network hybrid HJB equations in this paper, an optimal control viscosity theory for the closely related, yet distinct class of piecewise deterministic Markov processes has been developed in a series of papers (see $[17,18,19,11])$.

The paper is organized as follows: In section 2, we summarize the work developed in [14] where a class of the call admission and routing control problems for loss networks are formulated as optimal stochastic control problems and then the corresponding hybrid HJB equations are developed; in Section 3, the viscosity solutions to the hybrid HJB equations are analyzed and the continuity property of the value functions of the OSC problems are studied; Section 4 contains the main results of the paper, namely the uniqueness of viscosity solutions for this class of HJB equations under mild conditions; Section 5 constitutes the conclusion of the paper; and the state transition equation is given in Appendix A. 


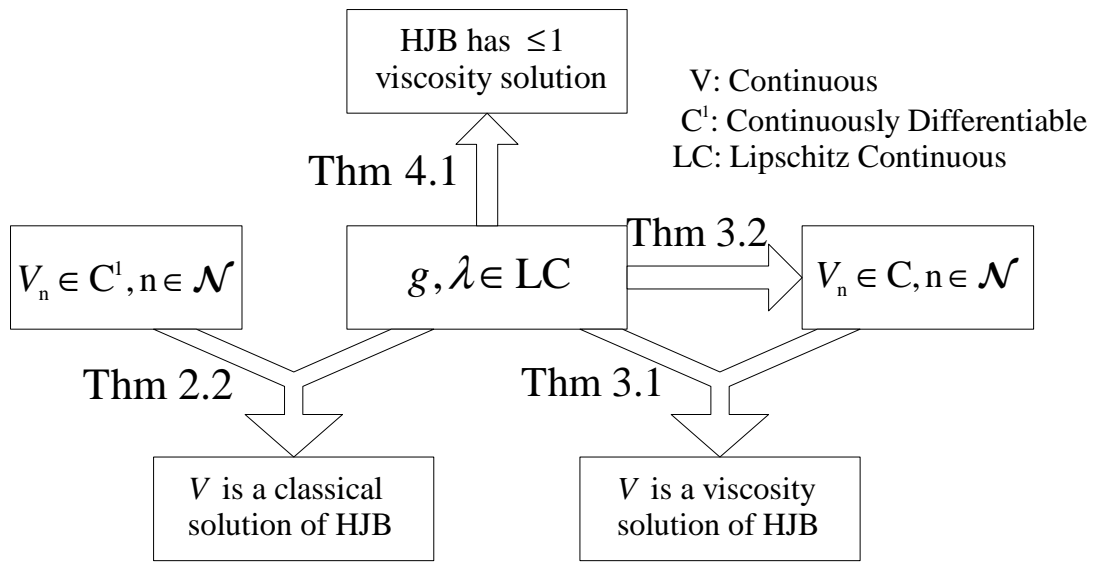

FIG. 1.1. The diagram of the main results

2. Optimal Control of Loss Networks. We summarize the main results developed in [14] where a class of control problems for loss networks have been formulated as optimal stochastic control problems which are implemented by solving the associated hybrid dynamic programming equations.

A loss network, which also was specified in [10], is a capacitated network $N$ et $(\mathbb{V}, \mathbb{L}$, $\mathbb{C})$ as formally defined below. Based upon this notion, the fundamental concept of a loss network system is formulated in Definition 2.6 below.

Definition 2.1. A loss network, $\operatorname{Net}(\mathbb{V}, \mathbb{L})$ consists of a set of vertices $\mathbb{V}=$ $\left\{v_{1}, \cdots, v_{V}\right\}$, with $V \in\{2,3, \cdots\}$, and a set of links $\mathbb{L}=\left\{l_{1}, \cdots, l_{L}\right\}, L \in \mathbb{Z}_{1}$, with $\mathbb{Z}_{1} \triangleq\{1,2, \cdots\}$, where each link $l \in \mathbb{L}$ is an ordered pair $\left(v^{\prime}, v^{\prime \prime}\right) \in \mathbb{V} \times \mathbb{V}$ of distinct vertices.

A loss network $N$ et $(\mathbb{V}, \mathbb{L})$ with (link) capacities

$$
\mathbb{C}=\left\{c_{s} \equiv c\left(l_{s}\right): 1 \leq s \leq L, c_{s} \in \mathbb{Z}_{1}\right\}
$$

shall be denoted by $\operatorname{Net}(\mathbb{V}, \mathbb{L}, \mathbb{C})$.

A route (or path), $r$ in the network $N$ et $(\mathbb{V}, \mathbb{L})$, connecting a vertex $o \in \mathbb{V}$ to a vertex $d \in \mathbb{V}$ is a finite sequence of vertices $r=\left(v_{1}^{\prime}, \cdots, v_{k}^{\prime}\right)$, such that

$$
v_{1}^{\prime}=o, v_{k}^{\prime}=d ; \quad v_{i}^{\prime} \neq v_{j}^{\prime}, \text { for } i \neq j ; \quad\left(v_{i}^{\prime}, v_{i+1}^{\prime}\right) \in \mathbb{L}, \text { for } i=1, \cdots, k-1 .
$$

The set of routes for all $\langle o, d\rangle$ pairs in the set $\mathbb{V} \triangle \subset \mathbb{V} \times \mathbb{V}$ in the network $N$ et $(\mathbb{V}, \mathbb{L})$ is denoted by $\mathcal{R}$, and we denote $R$ as the cardinality of $\mathcal{R}$, i.e. $R=|\mathcal{R}|$.

Notice: By abuse of notation, $r$ also denotes the indexing in $\mathcal{R}$, i.e. $r \in\{1, \cdots, R\}$.

The subset of routes with respect to a pair of vertices $\langle o, d\rangle$, denoted by $\mathcal{R}_{\langle o, d\rangle}$, is defined as $\mathcal{R}_{\langle o, d\rangle} \triangleq\left\{r=\left(v_{1}^{\prime}, \cdots, v_{k}^{\prime}\right) \in \mathcal{R} ; v_{1}^{\prime}=o, v_{k}^{\prime}=d\right\}$.

Fig.2.1 is an illustration of three distinct routes from $v_{1}$ to $v_{8}$, which are $\left(v_{1}, v_{2}, v_{5}\right.$, $\left.v_{4}, v_{8}\right),\left(v_{1}, v_{4}, v_{8}\right)$ and $\left(v_{1}, v_{3}, v_{7}, v_{8}\right)$ respectively in a loss network. 


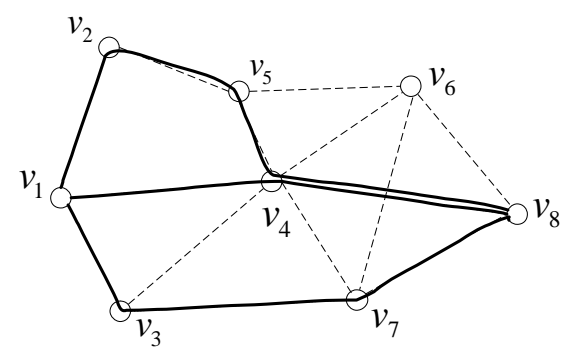

FIG. 2.1. Distinct Routes in A Loss Network

Definition 2.2. The (admissible) set of connection vectors, denoted $\mathcal{N}$, in $\mathcal{R}$ in the network with capacities $N e t(\mathbb{V}, \mathbb{L}, \mathbb{C})$, is defined as

$$
\mathcal{N}=\left\{\mathbf{n}=\left(n_{r}\right) \in \mathbb{Z}_{+}^{R}: \sum_{r \in \mathcal{R} ; l_{k} \in r} n_{r} \leq c_{k}, \forall k, 1 \leq k \leq L\right\}
$$

with $\mathbb{Z}_{+} \triangleq\{0,1, \cdots\}$, and $n_{r}$ denoting the number of active connections at route $r \in \mathcal{R}$.

Remark: In (2.2) for each fixed $l_{k}$ the set of $r \in \mathcal{R}$ appearing in the sum is the set of routes each of which contains $l_{k}$ as a link.

2.1. Loss Network Systems. A loss network system is a traffic structure specification and state transition equations superimposed on a loss network $N$ et $(\mathbb{V}, \mathbb{L}, C)$ as in Definition 2.1, whereby each of ordered pairs in $\mathbb{V}^{\triangle}$ defined as origin and destination, say $o$ and $d$, is associated with a random flow of call requests denoted $R q_{\langle o, d\rangle}^{+}$ to be considered first for network admission, and subsequent routing if admitted.

Call request flows between different $\langle o, d\rangle$ pairs are assumed to be stochastic processes of the renewal type, and are also assumed to be independent for distinct $\langle o, d\rangle$ pairs. When admitted, a call request is placed on an admissible route and becomes a connection. All connection durations are assumed to be independent identically distributed random variables. The above characterization is made precise in the defining assumptions below:

(S1) The probability space $(\Omega, \mathcal{F}, \mathbb{P})$ carries the family of independent stochastic processes and random variables $\left\{\mathrm{Rq}_{\langle o, d\rangle}^{+}, \eta_{m} ;\langle o, d\rangle \in \mathbb{V}^{\triangle}, m \in \mathbb{Z}_{1}\right\}$;

(S2) The call request process from $o$ towards $d, \mathrm{Rq}_{\langle o, d\rangle}^{+}: \mathbb{R}_{+} \times \Omega \rightarrow \mathbb{Z}_{+}$, is an autonomous renewal process with arrival rate equal to $\lambda_{\langle o, d\rangle}^{+}(s)$, where $s$ denotes the elapsed time since the most recent call request from $o$ to $d, e_{\langle o, d\rangle}^{+}$, event;

(S3) The duration of the $m$-th established connections for any $m \in \mathbb{Z}_{1}, \eta_{m}: \Omega \rightarrow$ $\mathbb{R}_{+}$, is a random variable with a general distribution $F\left(s^{\prime}\right)$ with a density $f\left(s^{\prime}\right)$ assumed to exist and where $s^{\prime}$ is the current age of the connection. Using conditional probabilities, this means that given that the connection has lasted $s^{\prime}$ so far, the instantaneous rate at which it will end is: $1 / \mu\left(s^{\prime}\right)=\frac{f\left(s^{\prime}\right)}{1-F\left(s^{\prime}\right)}$. 
In the framework of renewal processes, i.e. under assumptions (S1)-(S3), the state process $\mathbf{x}$ subject to a state dependent control law is a Markov process since the state in question is defined at time $t$ to comprise (see Definition 2.3 and Theorem 2.1):

(i) $\mathbf{n}$ the connection vector value, i.e. the vector summarizing the number of connections active on all admissible routes in the loss network;

(ii) $\zeta \equiv\left[\begin{array}{c}\psi \\ \varphi\end{array}\right]$ where $\psi \equiv\left[\begin{array}{c}\psi_{1} \\ \cdots \\ \psi_{|\mathbb{V} \triangle|}\end{array}\right]$ is a vector comprising the elapsed time from the last call request from $o$ to $d$ for all the $\langle o, d\rangle$ pairs in $\mathbb{V}^{\triangle}$ with some fixed ordering of these $\langle o, d\rangle$ pairs, herein called the waiting time vector; while $\varphi \equiv\left[\begin{array}{c}\varphi_{1,1} \\ \cdots \\ \varphi_{1, n_{1}} \\ \ldots \\ \varphi_{R, 1} \\ \cdots \\ \varphi_{R, n_{R}}\end{array}\right]$ is a vector comprising the age of each of the active connections on all routes $r \in \mathcal{R}$ with a fixed ordering of these routes and a chronological ordering of connections for a fixed route by arrival instant. $\varphi$ will be called the age vector.

Note that $\psi_{i}$ and $\varphi_{r, j}$ denote, respectively, (i) the elapsed time from the last call request from $o$ to $d$ with its ordering in $\mathbb{V}^{\triangle}$ equal to $i$, and (ii) the $j$-th active connection on route $r$; (iii) $e$ the (call request or connection ending) event.

The state space associated with the above description is formalized in Definition 2.3 below.

Definition 2.3. The state space, denoted $X, X \triangleq \dot{U}_{\mathbf{n} \in \mathcal{N}} X_{\mathbf{n}}$ with $X_{\mathbf{n}} \triangleq Z_{\mathbf{n}} \times E_{\mathbf{n}}$, where $\dot{U}$ denotes the disjoint union, $Z_{\mathbf{n}}$ and $E_{\mathbf{n}}$ denote respectively the pre-state space and (discrete) event space induced by a connection vector value $\mathbf{n}$, such that:

$$
Z_{\mathbf{n}}=\left\{z=\left[\begin{array}{c}
\mathbf{n} \\
\zeta
\end{array}\right] ; \zeta \in \mathbb{R}_{+}^{d(\mathbf{n})}\right\}, \quad d(\mathbf{n})=\left|\mathbb{V}^{\triangle}\right|+\Sigma_{r=1}^{R} n_{r},
$$

with $Z \triangleq \dot{\cup}_{\mathbf{n} \in \mathcal{N}} Z_{\mathbf{n}}$ and $\zeta=\left[\begin{array}{l}\psi \\ \varphi\end{array}\right] ;$ while

$$
E_{\mathbf{n}}=\left\{e_{\mathbf{n}}^{0}, e_{\langle o, d\rangle}^{+}, e_{r, j}^{-} ;\langle o, d\rangle \in \mathbb{V}^{\triangle}, r \in \mathcal{R}, j \in\left\{1, \cdots, n_{r}\right\}\right\}
$$

where $e_{\mathbf{n}}^{0}, e_{\langle o, d\rangle}^{+}$and $e_{r, j}^{-}$denote, respectively, (i) absence of a call request or a connection ending (event), (ii) a call request from $o$ to $d$, and (iii) the end of $j$ th active connection on route $r$.

Note that $e_{\mathbf{n}}^{0}$ is mapped to $\mathbf{0}$ with $\mathbf{0}$ as the zero vector in $\mathbb{R}^{d(\mathbf{n})} ; e_{\langle o, d\rangle}^{+}$is mapped to $a_{b}$ with $a_{b}$ as the $b$-th unit vector in $\mathbb{R}^{d(\mathbf{n})}$ with $b$ as the indexing number of the $\langle o, d\rangle$ pair in the set $\mathbb{V}^{\triangle}$; while $e_{r, j}^{-}$is mapped to $a_{\widehat{b}}$ with $\widehat{b}=\left|\mathbb{V}^{\triangle}\right|+\sum_{k=1}^{r-1} n_{k}+j$ 
as the position, in the vector $\zeta$, of the age variable associated with the $j$-th active connection on the $r$-th route.

Definition 2.4. The (admissible) control (value) set at a given state value $\mathbf{x}=(\mathbf{n}, \zeta, e) \in X$, denoted $U(\mathbf{x})$, is specified as:

$$
U(\mathbf{x})= \begin{cases}\{\mathbf{0}\} \cup\left\{a_{r} ; r \in \mathcal{R}_{\langle o, d\rangle}, \mathbf{n}+a_{r} \in \mathcal{N}\right\}, & \text { in case } e=e_{\langle o, d\rangle}^{+} \\ \left\{-a_{r}\right\}, & \text { in case } e=e_{r, j}^{-}, j=1, \cdots, n_{r}, \\ \{\mathbf{0}\}, & \text { otherwise }\end{cases}
$$

where $a_{r}$ denotes the $r$-th unit vector of $\mathbb{R}^{R}$ and we denote $U^{+}=\left\{a_{r} ; r=1, \cdots, R\right\}$.

Interpretation of (2.5): (i) $u\left(\mathbf{n}, \zeta, e_{\langle o, d\rangle}^{+}\right)=\mathbf{0}$ or $a_{r}$ denote respectively that the call request $e_{\langle o, d\rangle}^{+}$is rejected, or admitted and a new connection is established on route $r$, thus increasing $n_{r}$ by 1 ; while (ii) $u\left(\mathbf{n}, \zeta, e_{r, j}^{-}\right)=-a_{r}$ denotes the departure of the $j$-th connection on route $r$, thus decreasing $n_{r}$ by one. Note that the change in the connection vector $\mathbf{n}$ will be accompanied by a restructuring of the vector $\zeta$, and a relabeling of the events in the $e$ point process. These changes are described in the transition equations (2.9) and (2.10) below.

$\mathbf{z}$ and $e$ necessarily depend progressively on $(\Omega, \mathcal{F}, \mathbb{P})$ with $\mathcal{F}_{t}=\sigma\left(z_{s^{-}}, e_{s} ; s \in\right.$ $[0, t]) \in \mathcal{F}$. We refer to $\mathbf{z}=\{\mathbf{z}(t, \omega) ; t \in[0, T], \omega \in \Omega\}$, and $e=\{e(t, \omega) ; t \in[0, T], \omega \in$ $\Omega\}$ as pre-state and event processes; and refer to $\mathbf{x}$ as the state process, such that

$$
\mathbf{x}(t, \omega) \triangleq\left(\mathbf{z}\left(t^{-}, \omega\right), e(t, \omega)\right), \text { for all } t \in[0, T], \omega \in \Omega,
$$

with $\mathbf{z}\left(t^{-}, \omega\right)=\lim _{s \uparrow t} \mathbf{z}(s, \omega)$.

The set of (admissible state dependent) control laws with the interval $[0, T]$, is denoted by $\mathcal{U}[0, T]$, and is given by,

$$
\mathcal{U}[0, T]=\left\{u:[0, T] \times X \rightarrow U ; \quad u_{t} \text { is } \sigma\left(\mathbf{x}_{t}\right) \text { measurable, } t \in[0, T]\right\}
$$

We term a sequence of event instants $\left\{t_{j}(\omega)\right\}$ in $[0, \infty)$

$$
0 \leq t_{1}(\omega) \leq \cdots \leq t_{j}(\omega) \leq \cdots, \quad \omega \in \Omega
$$

at which call request or connection ending event occurs as a sequence of event instants t. $: \mathbb{Z}_{+} \times \Omega \rightarrow \mathbb{R}_{+}$.

Definition 2.5. (State Transition Equation) 
Subject to an control law $u \in \mathcal{U}[0, T]$ the event and pre-state transition equations of the $u$-controlled state process $\mathbf{x}^{u}$ are given respectively as follows:

$$
e_{t}^{u}=\left\{\begin{array}{ll}
e_{\langle j, k\rangle}^{+}, & \text {in case } R q_{\langle j, k\rangle}^{+}(t)=R q_{\langle j, k\rangle}^{+}\left(t^{-}\right)+1, \text { for some }\langle o, d\rangle \text { pair in } \mathbb{V}^{\triangle} \\
e_{r, j}^{-}, & \text {in case } \eta_{m}=\varphi_{r, j}(t), \text { for some } j \in\left\{1, \cdots, n_{r}\left(t^{-}\right)\right\} \\
e_{\mathbf{n}}^{0}, & \text { otherwise }
\end{array},\right.
$$

such that the $j$-th active connection on route $r$ is the $m$-th established connection on the whole network system.

$$
\mathbf{z}_{t}^{u}= \begin{cases}\mathbf{z}_{t^{-}}^{u} \star u_{t} \equiv\left[\begin{array}{c}
\mathbf{n}_{t^{-}}^{u}+u_{t} \\
\zeta_{t^{-}}^{u} \circ u_{t}
\end{array}\right], & \text { in case } t=t_{i} \\
{\left[\begin{array}{c}
\mathbf{n}_{t_{i-1}} \\
\zeta_{t_{i-1}}+\left(t-t_{i-1}\right) 1_{\zeta_{t_{i-1}}}
\end{array}\right],} & \text { in case } t_{i-1}<t<t_{i}\end{cases}
$$

where (i) $\zeta_{t^{-}}^{u} \circ u_{t} \triangleq A_{t}\left(I_{d\left(\mathbf{n}_{t^{-}}\right)}-e_{t} e_{t}^{\prime}\right) \zeta_{t^{-}}$with the matrix $A_{t}$ specified in (2.11) below, (ii) $e^{\prime}$ denotes the transposition of vector $e$, (iii) $0_{\mathbf{n}} \equiv(0, \cdots, 0)^{\prime} \in \mathbb{Z}_{+}^{R}$ and $1_{\zeta} \equiv(1, \cdots, 1)^{\prime}$, with the dimension of $\zeta$ varies in accordance with $A$.

$$
\begin{gathered}
A_{t} \equiv A\left(\mathbf{n}_{t^{-}}^{u}, e_{t}^{u}, u_{t}\right)= \begin{cases}A^{+}\left(\mathbf{n}_{t^{-}}^{u} ; r\right), & \text { in case } u_{t}=a_{r} \\
A^{-}\left(\mathbf{n}_{t^{-}}^{u} ; r, k\right), & \text { in case } e_{t}^{u}=e_{r, k}^{-}, u_{t}=-a_{r}, \text { such that } \\
I_{D}, & \text { otherwise }\end{cases} \\
A^{+}\left(\mathbf{n}_{t^{-}}^{u} ; r\right)=\left[\begin{array}{cr}
I_{m} & 0_{(m+1) \times(d-m)} \\
0_{(d-m+1) \times m} & I_{(d-m)}
\end{array}\right], \quad \text { with } m=\left|\mathbb{V}^{\Delta}\right|+\sum_{j=1}^{r} n_{j}^{u}\left(t^{-}\right), \\
A^{-}\left(\mathbf{n}_{t^{-}}^{u} ; r, k\right)=\left[\begin{array}{rr}
I_{\widehat{m}} & 0_{\widehat{m} \times(d-\widehat{m})} \\
0_{(d-\widehat{m}-1) \times(\widehat{m}+1)} & I_{(d-\widehat{m}-1)}
\end{array}\right], \\
\text { with } \widehat{m}=\left|\mathbb{V}^{\Delta}\right|+\sum_{j=1}^{r-1} n_{j}^{u}\left(t^{-}\right)+(k-1),
\end{gathered}
$$

where $I_{h}$ and $0_{h \times j}$ denote the $h$-dimension identity matrix and $h \times j$-dimension zero matrix respectively. Recall that $d(\mathbf{n})$ is specified in (2.3).

Interpretation of (2.11): In simple terms, (i) when $u_{t}=a_{r}$ set to zero the waiting time variable associated with the $\langle o, d\rangle$ pair corresponding to the admitted call request, create a new zero age entry for the newly created connection at position $\Sigma_{i=1}^{r} n_{i}$ within the age vector, and shift down by one the positions of connection ages associated with routes $k>r$ within in the age vector; (ii) for $u_{t}=0$, once again, the waiting time variable associated with the $\langle o, d\rangle$ pair corresponding to the call request just rejected is set to zero, while the age vector is left unchanged; (iii) finally for $u_{t}=-a_{r}$, the 
dimension of the age vector is reduced by one, and positions of all age variables associated with routes numbered $k$ for $k>r$ are shifted by one up within the age vector.

Definition 2.6. ((Stochastic) Loss Network System)

A stochastic loss network system is a loss network $N$ et $(\mathbb{V}, \mathbb{L}, C)$, over which one has superimposed a traffic structure specified by assumptions (S1)-(S3), and which has been associated with a family of admissible state dependent control laws as in (2.7) and a state transition equation as in (2.9) and (2.10).

Theorem 2.1. Under assumptions (S1)-(S3) and subject to a control law $u \in$ $\mathcal{U}[0, T]$, the state process $\mathbf{x}^{u}$ is a Markov process. Furthermore, in case $u$ is time shift invariant, $\mathbf{x}^{u}$ is a homogeneous Markov process.

The proof of Theorem 2.1 is similar to that of Theorem 25.5 in [3]; see [12, 14] for the details.

2.2. The Optimal Control of Stochastic Loss Networks. Call admission and routing problems for loss networks can be formulated as optimal stochastic control problems which necessarily require the specifications of (i) the state dynamics, and (ii) an integrated cost function covering a given interval.

Definition 2.7. ((State dependent) optimal stochastic control problems for finite horizon loss network systems)

For any $s \in[0, T)$ and the pre-state value at $s$ as $\mathbf{z}_{s}$, we assume that the (expected integrated) cost function subject to any (state dependent) control law $u \in \mathcal{U}[s, T]$, is specified as follows:

$$
J\left(s, \mathbf{z}_{s} ; u\right)=\mathbb{E}_{\mid \mathbf{z}_{s}}\left\{\int_{s}^{T} g(t, \mathbf{z}(t)) d t\right\}
$$

where the loss function $g$ is bounded and measurable with respect to $(t, \mathbf{z})$.

The optimal stochastic control (OSC) problem (or family of OSC problems) subject to state dependent control laws) is given by the infimization:

$$
V_{\mathbf{n}_{s}}\left(s, \zeta_{s}\right) \equiv V\left(s, \mathbf{z}_{s}\right)=\inf _{u \in \mathcal{U}[s, T]} J\left(s, \mathbf{z}_{s} ; u\right)
$$

where the collection of indexed functions $V \equiv\left\{V_{\mathbf{n}}:[s, T] \times \mathbb{R}_{+}^{d(\mathbf{n})} \rightarrow \mathbb{R} ; \mathbf{n} \in \mathcal{N}\right\}$ is called the value function of the family OSC problems. In case an infimizing function $u^{0} \in \mathcal{U}[s, T]$ exists, $u^{0}$ shall be called an optimal control law for the OSC problem.

For any $\mathbf{n} \in \mathcal{N}$ and $\left(s_{1}, \zeta_{1}\right),\left(s_{2}, \zeta_{2}\right) \in[0, T] \times \mathbb{R}_{+}^{d(\mathbf{n})}$, with $d(\mathbf{n})$ specified in (2.3), 
we define

$$
\|(s, \zeta)-(\widehat{s}, \widehat{\zeta})\| \triangleq \sqrt{(s-\widehat{s})^{2}+\sum_{i=1}^{d(\mathbf{n})}\left(\zeta^{i}-\widehat{\zeta}^{i}\right)^{2}}
$$

where $\zeta^{i}$ denotes the $i$-th component of the vector $\zeta$.

We assume the following assumptions:

(S4) For any event $e \in E_{\mathbf{n}}, \lambda^{e}(\zeta)$ is uniformly bounded and Lipschitz continuous, such that

$$
\begin{aligned}
& \sup _{\zeta \in \mathbb{R}_{+}^{d(\mathbf{n})}}\left(\sum_{e \in E_{\mathbf{n}}} \lambda^{e}(\zeta)\right)<L, \\
& \left|\lambda^{e}(\zeta)-\lambda^{e}(\widehat{\zeta})\right|<L\|\zeta-\widehat{\zeta}\|, \quad \text { for any } \zeta, \widehat{\zeta} \in \mathbb{R}_{+}^{d(\mathbf{n})}, \mathbf{n} \in \mathcal{N},
\end{aligned}
$$

where $\lambda^{e}(\zeta)=\left\{\begin{array}{ll}\lambda_{\langle o, d\rangle}^{+}\left(\psi_{\langle o, d\rangle}\right) & \text { in case } e=e_{\langle o, d\rangle}^{+} \\ \mu\left(\varphi_{r, j}\right) & \text { in case } e=e_{r, j}^{-} \text {with } j \in\left\{1, \cdots, n_{r}\right\}\end{array} ;\right.$

(S5) The loss function $g$ is uniformly bounded, such that

$$
\left|g_{\mathbf{n}}(t, \zeta)\right| \leq L, \quad \text { for any }(t, \mathbf{n}, \zeta) \in[0, T] \times Z,
$$

and, for all $\mathbf{n} \in \mathcal{N}, g_{\mathbf{n}}$ is uniformly Lipschitz continuous with respect to $(t, \zeta)$, such that for any $t, s \in[0, T]$ and $\zeta, \widehat{\zeta} \in \mathbb{R}_{+}^{d(\mathbf{n})},\left|g_{\mathbf{n}}(t, \zeta)-g_{\mathbf{n}}(s, \widehat{\zeta})\right|<$ $L\|(t, \zeta)-(s, \widehat{\zeta})\|$.

Remark: The specifications (S4) and (S5) with an identical finite positive valued $L$ are equivalent to those with distinct respectively finite positive values.

TheOREM 2.2. (Hybrid HJB Equations for network OSC Problems)

Suppose $V_{\mathbf{n}} \in C^{1}\left([0, T] \times \mathbb{R}_{+}^{d(\mathbf{n})}\right)$ for all $\mathbf{n} \in \mathcal{N}$. Then under assumptions (S1)-(S5), $V$ is a solution of collection of coupled partial differential equations below:

$$
0=\left(\frac{\partial}{\partial t}+\sum_{i=1}^{d(\mathbf{n})} \frac{\partial}{\partial \zeta^{i}}\right) V_{\mathbf{n}}(t, \zeta)+\sum_{e \in E_{\mathbf{n}}} \lambda^{e}(\zeta)\left(\min _{u_{t} \in U} V_{\mathbf{n}+u_{t}}\left(t, \zeta \circ u_{t}\right)-V_{\mathbf{n}}(t, \zeta)\right)+g_{\mathbf{n}}(t, \zeta),
$$

with the boundary condition $V_{\mathbf{n}}(T, \zeta)=0$, for all $(\mathbf{n}, \zeta) \in Z$, where $\zeta^{i}$ denotes the $i$-th component of the vector $\zeta$ and the operator $\zeta \circ u$ is specified in $(2.10)$.

Proof. Under assumptions (S4) and (S5), we obtain that the event rate $\lambda$ and loss function $g$ are bounded and continuous from the right respectively, then together with assumptions (S1)-(S3), by Theorem 3.4 in [14], we get the conclusion.

\section{Viscosity Solutions for Hybrid HJB Equations.}

Definition 3.1. The collection of functions $v=\left\{v_{\mathbf{n}} \in C\left([0, T] \times \Sigma_{\mathbf{n}}\right) ; \mathbf{n} \in \mathcal{N}\right\}$ with the boundary condition $v_{\mathbf{n}}(T, \zeta) \leq 0$, for all $(\mathbf{n}, \zeta) \in Z$, is called a viscosity 
subsolution of the hybrid HJB equation (2.17), if for any collection of continuously differentiable functions $\phi=\left\{\phi_{\mathbf{n}} \in C^{1}\left([0, T] \times \Sigma_{\mathbf{n}}\right) ; \mathbf{n} \in \mathcal{N}\right\}$, whenever

(1) $v_{\mathbf{n}}-\phi_{\mathbf{n}}$ attains a local maximum at $(t, \zeta) \in[0, T] \times \Sigma_{\mathbf{n}}$, with $v_{\mathbf{n}}(t, \zeta)-\phi_{\mathbf{n}}(t, \zeta)=$ 0

(2) $\phi_{\mathbf{n}+u_{t}}\left(t, \zeta \circ u_{t}\right)=v_{\mathbf{n}+u_{t}}\left(t, \zeta \circ u_{t}\right)$ subject to all $u_{t} \in U(z, e)$ for any $e \in E_{\mathbf{n}}$, the following inequality holds

$$
0 \leq\left(\frac{\partial}{\partial t}+\sum_{i=1}^{d(\mathbf{n})} \frac{\partial}{\partial \zeta^{i}}\right) \phi_{\mathbf{n}}(t, \zeta)+g(t, z)+\sum_{e \in E_{\mathbf{n}}} \lambda^{e}(\zeta)\left(\min _{u_{t} \in U} \phi_{\mathbf{n}+u_{t}}\left(t, \zeta \circ u_{t}\right)-\phi_{\mathbf{n}}(t, \zeta)\right) .
$$

$v$ is called a viscosity supersolution of the hybrid HJB equation (2.17) under the conditions above where " $\leq$ " and "local maximum" are substituted by " $\geq$ " and "local minimum" respectively.

$v$ is called a viscosity solution of the hybrid HJB equation (2.17), if $v$ is both a viscosity subsolution and supersolution of (2.17).

It may be verified that the viscosity subsolution and supersolution for the hybrid HJB equation (2.17) given in Definition 3.2 below are respectively equivalent to those given in Definition 3.1.

Definition 3.2. The collection of functions $v=\left\{v_{\mathbf{n}} \in C\left([0, T] \times \Sigma_{\mathbf{n}}\right) ; \mathbf{n} \in \mathcal{N}\right\}$ with the boundary condition $v_{\mathbf{n}}(T, \zeta) \leq 0$, for all $(\mathbf{n}, \zeta) \in Z$, is called a viscosity subsolution of the hybrid HJB equation (2.17), if for any collection of continuously differentiable functions $\phi=\left\{\phi_{\mathbf{n}} \in C^{1}\left([0, T] \times \Sigma_{\mathbf{n}}\right) ; \mathbf{n} \in \mathcal{N}\right\}$, for any $\mathbf{n} \in \mathcal{N}$, whenever $v_{\mathbf{n}}-\phi_{\mathbf{n}}$ attains a local maximum at $(t, \zeta) \in[0, T] \times \Sigma_{\mathbf{n}}$, we have

$$
0 \leq\left(\frac{\partial}{\partial t}+\sum_{i=1}^{d(\mathbf{n})} \frac{\partial}{\partial \zeta^{i}}\right) \phi_{\mathbf{n}}(t, \zeta)+g_{\mathbf{n}}(t, \zeta)+\sum_{e \in E_{\mathbf{n}}} \lambda^{e}(\zeta)\left(\min _{u_{t} \in U} v_{\mathbf{n}+u_{t}}\left(t, \zeta \circ u_{t}\right)-v_{\mathbf{n}}(t, \zeta)\right)
$$

$v$ is called a viscosity supersolution of (2.17) under the conditions above where " $\leq$ " and "local maximum" are substituted by " $\geq$ " and "local minimum" respectively.

Theorem 3.1. Provided the value function $V=\left\{V_{\mathbf{n}} \in C\left([0, T] \times \mathbb{R}_{+}^{d(\mathbf{n})}\right) ; \mathbf{n} \in \mathcal{N}\right\}$ is continuous, it will be a viscosity solution of (2.17).

Proof. Consider any collection of functions $\phi=\left\{\phi_{\mathbf{n}}:[0, T] \times \mathbb{R}_{+}^{d(\mathbf{n})} \rightarrow \mathbb{R} ; \mathbf{n} \in \mathcal{N}\right\}$, such that $\phi_{\mathbf{n}} \in C^{1}\left([0, T] \times \mathbb{R}_{+}^{d(\mathbf{n})}\right)$, for all $\mathbf{n} \in \mathcal{N}$, and assume furthermore that

(1) $V_{\mathbf{n}}-\phi_{\mathbf{n}}$ attains the local maximum at $(t, \zeta)$, with $\phi_{\mathbf{n}}(t, \zeta)=V_{\mathbf{n}}(t, \zeta)$;

(2) $\phi_{\mathbf{n}+u_{t}}\left(t, \zeta \circ u_{t}\right)=V_{\mathbf{n}+u_{t}}\left(t, \zeta \circ u_{t}\right)$ subject to all $u_{t} \in U(z, e)$ for any $e \in E_{\mathbf{n}}$. Then by Definition 3.1, $V$ is a viscosity subsolution of (2.17) if the inequality (3.1) holds at $(t, \mathbf{n}, \zeta)$.

Let $t+\tau(\omega)$ denote the first event instant after instant $t$; then there exists a random variable $\theta$, such that $0 \leq \theta(\omega)<\tau(\omega)$ a.s., which implies $\mathbf{n}_{t+\theta}=\mathbf{n}$, and by 
the local maximality assumed in (1) above, for some small enough $\theta$, we have

$$
V_{\mathbf{n}}\left(t+\theta, \zeta+\theta \mathbf{1}_{\zeta}\right)-\phi_{\mathbf{n}}\left(t+\theta, \zeta+\theta \mathbf{1}_{\zeta}\right) \leq V_{\mathbf{n}}(t, \zeta)-\phi_{\mathbf{n}}(t, \zeta),
$$

with $\mathbf{1}_{\zeta}=(1 ; 1 ; \cdots ; 1) \in \mathbb{R}^{D}$.

Hence for any $u \in \mathcal{U}[t, t+\theta]$, we obtain that

$$
\begin{array}{rlr} 
& \mathbb{E}\left\{V_{\mathbf{n}_{t+\theta}}\left(t+\theta, \zeta+\theta \mathbf{1}_{\zeta}\right)\right\}-V_{\mathbf{n}}(t, \zeta)=\mathbb{E}\left\{V_{\mathbf{n}_{t+\theta}}\left(t+\theta, \zeta+\theta \mathbf{1}_{\zeta}\right)-V_{\mathbf{n}}(t, \zeta)\right\} \\
\leq & \mathbb{E}\left\{\phi_{\mathbf{n}_{t+\theta}}\left(t+\theta, \zeta+\theta \mathbf{1}_{\zeta}\right)-\phi_{\mathbf{n}}(t, \zeta)\right\}, & \text { by the inequality }(3.3) \\
(3.4)= & \mathbb{E}\left\{\int_{t}^{t+\theta} \mathcal{A} \phi_{\mathbf{n}_{\nu}}\left(\nu, \zeta_{\nu}\right) d \nu\right\}, & \text { by the Dynkin formula }
\end{array}
$$

where $\mathcal{A} \phi_{\mathbf{n}}(t, \zeta)=\left(\frac{\partial}{\partial t}+\sum_{i=1}^{d(\mathbf{n})} \frac{\partial}{\partial \zeta^{i}}\right) \phi_{\mathbf{n}}(t, \zeta)+\sum_{e \in E_{\mathbf{n}}} \lambda^{e}(\zeta)\left(\phi_{\mathbf{n}+u_{t}}\left(t, \zeta \circ u_{t}\right)-\phi_{\mathbf{n}}(t, \zeta)\right)$.

Also, by the principle of optimality, we obtain that

$$
V_{\mathbf{n}}(t, \zeta) \leq \mathbb{E}\left\{\int_{t}^{t+\theta(\omega)} g\left(\nu, z_{\nu}\right) d \nu+V_{\mathbf{n}_{t+\theta}}\left(t+\theta, \zeta+\theta \mathbf{1}_{\zeta}\right)\right\}
$$

Then (3.5) together with inequality (3.4), we lead to:

$$
0 \leq \mathbb{E}\left\{\int_{t}^{t+\theta(\omega)}\left(g\left(\nu, z_{\nu}\right)+\mathcal{A} \phi_{\mathbf{n}_{\nu}}\left(\nu, \zeta_{\nu}\right)\right) d \nu\right\}
$$

Hence with $\theta(\omega) \rightarrow 0$, by (3.6) and the assumption (2) above, we obtain that $V$ is a viscosity subsolution of the hybrid HJB equation (2.17). Similarly one may prove that $V$ is also a viscosity supersolution of (2.17).

Theorem 3.2. Under assumptions (S1)-(S5), the value function $V$ will be continuous, i.e. $V_{\mathbf{n}} \in C\left([0, T] \times \mathbb{R}_{+}^{d(\mathbf{n})}\right)$, for any $\mathbf{n} \in \mathcal{N}$.

Proof. See Proposition 3.1 in Section 3, [15] for the proof.

Proposition 3.1. Under assumptions (S1)-(S5), the value function $V$ is a viscosity solution of the hybrid HJB equation (2.17).

Proof. The proof is straightforward based upon Theorems 3.1 and 3.2.

4. Uniqueness of Viscosity Solutions of Hybrid HJB Equations (2.17). Lemma 4.1. For any $\mathbf{n} \in \mathcal{N}, e \in E_{\mathbf{n}}$, and $u \in U(\mathbf{n}, e)$, the map ou $: \mathbb{R}_{+}^{d(\mathbf{n})} \rightarrow$ $\mathbb{R}_{+}^{d(\mathbf{n}+u)}$, (please refer to state transition equation (2.10)) is a contraction map (or non-expansive) with the contractivity coefficient 1, i.e.

$$
\|\zeta \circ u-\widehat{\zeta} \circ u\| \leq\|\zeta-\widehat{\zeta}\|, \quad \forall \zeta, \widehat{\zeta} \in \mathbb{R}_{+}^{d(\mathbf{n})}
$$

The proof is straightforward by the $u$-controlled state transition equation specified in $(2.10)$.

We prove the uniqueness of viscosity solutions of (2.17) by assuming there exist two distinct viscosity solutions and then proceeding in three steps: 
(Step 1) We use the postulated multiple viscosity solutions to construct a comparison (vector) function $\Phi$ depending on some strictly positive parameters $\varepsilon, \alpha$, $\beta$ and $\gamma$ to be defined below, and which is a perturbation of the difference function between the posited distinct viscosity solutions; then show that $\Phi$ possesses an interior global maximum;

(Step 2) Using the defining properties of viscosity solutions of the HJB equation (2.17), we establish a set of inequalities at the above fixed parameterized interior global maximum;

(Step 3) By letting the positive parameters, $\alpha$ and $\beta$, to go to zero, in the inequalities established in Step 2 above, we get a contradiction thus establishing uniqueness of viscosity solutions.

(Step 1) Hypothesize two distinct viscosity solutions $v, \widehat{v}$ of the HJB equation (2.17) and construct a comparison function $\Phi$ and show that $\Phi$ possesses an interior global maximum.

For any $\mathbf{n} \in \mathcal{N}$, suppose that $v_{\mathbf{n}}, \widehat{v}_{\mathbf{n}}:[0, T] \times \mathbb{R}_{+}^{d(\mathbf{n})} \rightarrow \mathbb{R}$ are two viscosity solutions of (2.17). To prove the uniqueness of viscosity solutions of (2.17), it suffices to prove the symmetric property

$$
v_{\mathbf{n}}(s, \zeta) \leq \widehat{v}_{\mathbf{n}}(s, \zeta), \quad \text { for all } \mathbf{n} \in \mathcal{N} \quad \text { and } \quad \text { for all }(s, \zeta) \in[0, T] \times \mathbb{R}_{+}^{d(\mathbf{n})}
$$

Suppose that the above is not true, then there exists some $\widehat{\mathbf{n}} \in \mathcal{N},(\widehat{s}, \widehat{\zeta}) \in$ $[0, T] \times \mathbb{R}_{+}^{d(\widehat{\mathbf{n}})}$, such that $v_{\widehat{\mathbf{n}}}(\widehat{s}, \widehat{\zeta})-\widehat{v}_{\widehat{\mathbf{n}}}(\widehat{s}, \widehat{\zeta})>0$

Hence, by the continuity property of $v_{\widehat{\mathbf{n}}}$ and $\widehat{v}_{\widehat{\mathbf{n}}}$, there exists $T_{0}, 0<T_{0}<T$, such that

$$
\sup _{(s, \zeta) \in \mathbb{N}\left(\widehat{\mathbf{n}}, T_{0}\right)}\left\{v_{\widehat{\mathbf{n}}}(s, \zeta)-\widehat{v}_{\widehat{\mathbf{n}}}(s, \zeta)\right\}>0
$$

where

$$
\mathbb{N}\left(\widehat{\mathbf{n}}, T_{0}\right) \triangleq\left\{(t, \zeta) \in\left(T-T_{0}, T\right) \times \mathbb{R}_{+}^{d(\widehat{\mathbf{n}})} \mid\|\zeta\|<L_{0}\left[t-T+T_{0}\right]\right\},
$$

with $L_{0}=D T /\left(T-T_{0}\right), D=\max \left\{\sqrt{d(\mathbf{n})+1} ; \quad \zeta \in \mathbb{R}_{+}^{d(\mathbf{n})}, \mathbf{n} \in \mathcal{N}\right\}$, and $\|\zeta\|=$ $\sqrt{\sum_{i=1}^{d(\mathbf{n})}\left(\zeta^{i}\right)^{2}}, \quad$ for all $\zeta \in \mathbb{R}_{+}^{d(\mathbf{n})}$, where $\zeta^{i}$ denote the $i$-th component of vector $\zeta$.

By (4.3), there exists a strictly positive real number $\bar{\gamma}$, such that

$$
\max _{\mathbf{n} \in \mathcal{N}}\left\{\sup _{(s, \zeta) \in \mathbb{N}\left(\mathbf{n}, T_{0}\right)}\left\{v_{\mathbf{n}}(s, \zeta)-\widehat{v}_{\mathbf{n}}(s, \zeta)\right\}\right\} \geq \bar{\gamma}>0
$$

We consider $\varepsilon, \delta>0$, such that $\varepsilon+\delta<L_{0} T_{0}$. Take $K>0$ such that

$$
K>\sup _{\left(\mathbf{n}, t, \zeta_{1}, s, \zeta_{2}\right) \in \mathcal{N} \times \mathbb{N} \times \mathbb{N}}\left\{v_{\mathbf{n}}\left(t, \zeta_{1}\right)-\widehat{v}_{\mathbf{n}}\left(s, \zeta_{2}\right)\right\},
$$


where

$$
\mathcal{N} \times \mathbb{N} \times \mathbb{N} \triangleq\left\{\left(\mathbf{n}, t, \zeta_{1}, s, \zeta_{2}\right) \mid \mathbf{n} \in \mathcal{N},\left(t, \zeta_{1}, s, \zeta_{2}\right) \in \mathbb{N}\left(\mathbf{n}, T_{0}\right) \times \mathbb{N}\left(\mathbf{n}, T_{0}\right)\right\} .
$$

We define a function $h: \mathbb{R} \rightarrow \mathbb{R}$, such that $h \in C^{\infty}(-\infty, \infty)$, (see Figure 4.1)

$$
h(t)=\left\{\begin{array}{ll}
0, & t \leq-\delta, \\
-K, & t \geq 0,
\end{array} \quad \text { and } \quad \frac{d h(t)}{d t} \leq 0, \text { for all } t \in \mathbb{R}\right.
$$

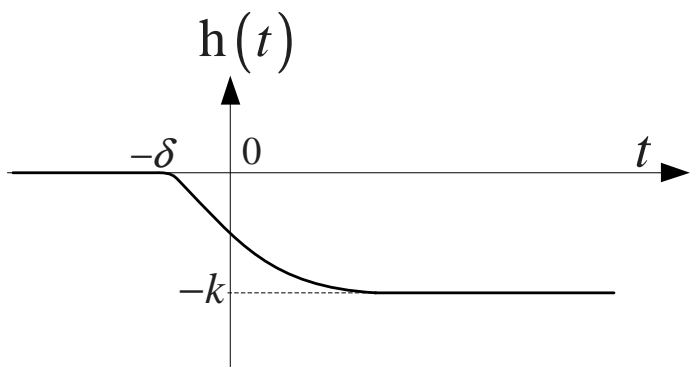

FIG. 4.1. The function $h$

For any $\alpha, \beta, \gamma>0$, we define a collection of comparison functions $\Phi=\left\{\Phi_{\mathbf{n}}\right.$ : $\left.\mathbb{N}\left(\mathbf{n}, T_{0}\right) \times \mathbb{N}\left(\mathbf{n}, T_{0}\right) \rightarrow \mathbb{R} ; n \in \mathcal{N}\right\}$, such that

$$
\begin{aligned}
& \Phi_{\mathbf{n}}\left(t, \zeta_{1}, s, \zeta_{2}\right)=v_{\mathbf{n}}\left(t, \zeta_{1}\right)-\widehat{v}_{\mathbf{n}}\left(s, \zeta_{2}\right)-\frac{1}{\alpha}\left\|\zeta_{1}-\zeta_{2}\right\|^{2}-\frac{1}{\beta}[t-s]^{2} \\
& \quad+h\left(\left\langle\zeta_{1}\right\rangle_{\varepsilon}-L_{0}\left[t-T+T_{0}\right]\right)+h\left(\left\langle\zeta_{2}\right\rangle_{\varepsilon}-L_{0}\left[t-T+T_{0}\right]\right)+\gamma[t+s]-2 \gamma T,
\end{aligned}
$$

where $\langle\zeta\rangle_{\varepsilon} \triangleq \sqrt{\|\zeta\|^{2}+\varepsilon^{2}}$, for all $\zeta \in \mathbb{R}_{+}^{d(\mathbf{n})}$.

Since, for any $\mathbf{n} \in \mathcal{N}, \Phi_{\mathbf{n}}$ is continuous and the closed set of $\mathbb{N}\left(\mathbf{n}, T_{0}\right) \times \mathbb{N}\left(\mathbf{n}, T_{0}\right)$, denoted $\overline{\mathbb{N}\left(\mathbf{n}, T_{0}\right) \times \mathbb{N}\left(\mathbf{n}, T_{0}\right)}$ is compact, $\Phi$ shall attain its maximum over $\overline{\mathcal{N} \times \mathbb{N} \times \mathbb{N}}$ at $\left(\mathbf{n}_{0}, t_{0}, \zeta_{1}^{0}, s_{0}, \zeta_{2}^{0}\right)$ which depends upon the parameters $(\alpha, \beta, \gamma, \varepsilon, \delta)$, where $\overline{\mathcal{N}} \times \mathbb{N} \times \mathbb{N}$ is specified as

$$
\overline{\mathcal{N} \times \mathbb{N} \times \mathbb{N}}=\left\{\left(\mathbf{n}, t, \zeta_{1}, s, \zeta_{2}\right) \mid \mathbf{n} \in \mathcal{N},\left(t, \zeta_{1}, s, \zeta_{2}\right) \in \overline{\mathbb{N}\left(\mathbf{n}, T_{0}\right) \times \mathbb{N}\left(\mathbf{n}, T_{0}\right)}\right\}
$$

At this point we need the following lemma.

Lemma 4.2. For any $\alpha, \beta, \gamma, \varepsilon, \delta>0$, such that $\varepsilon+\delta<L_{0} T_{0}$, we have the maximum point $\left(\mathbf{n}_{0}, t_{0}, \zeta_{1}^{0}, s_{0}, \zeta_{2}^{0}\right)$ satisfies the following:

$$
\left\|\zeta_{1}^{0}\right\|<L_{0}\left[t_{0}-T+T_{0}\right] \quad \text { and } \quad\left\|\zeta_{2}^{0}\right\|<L_{0}\left[s_{0}-T+T_{0}\right]
$$

Proof. Suppose that the inequalities (4.11) do not hold, then

$$
\left\|\zeta_{1}^{0}\right\| \geq L_{0}\left[t_{0}-T+T_{0}\right] \quad \text { or } \quad\left\|\zeta_{2}^{0}\right\| \geq L_{0}\left[s_{0}-T+T_{0}\right]
$$


Then by (4.12) and $\langle\zeta\rangle_{\varepsilon}=\sqrt{\|\zeta\|^{2}+\varepsilon^{2}}>\|\zeta\|$, for all $\varepsilon>0$, we obtain that

$$
\left\langle\zeta_{1}^{0}\right\rangle_{\varepsilon}-L_{0}\left[t_{0}-T+T_{0}\right]>0 \quad \text { or } \quad\left\langle\zeta_{2}^{0}\right\rangle_{\varepsilon}-L_{0}\left[s_{0}-T+T_{0}\right]>0
$$

hence by the definition of function $h$, we get that

$$
h\left(\left\langle\zeta_{1}^{0}\right\rangle_{\varepsilon}-L_{0}\left[t_{0}-T+T_{0}\right]\right)+h\left(\left\langle\zeta_{2}^{0}\right\rangle_{\varepsilon}-L_{0}\left[s_{0}-T+T_{0}\right]\right) \leq-K
$$

Also, since $\left(\mathbf{n}_{0}, t_{0}, \zeta_{1}^{0}, s_{0}, \zeta_{2}^{0}\right)$ is a maximum point in $\overline{\mathcal{N} \times \mathbb{N} \times \mathbb{N}}$, we have

$$
\Phi_{\mathbf{n}_{0}}(T, 0, T, 0) \leq \Phi_{\mathbf{n}_{0}}\left(t_{0}, \zeta_{1}^{0}, s_{0}, \zeta_{2}^{0}\right)
$$

Hence, by (4.14) and the specifications of $K$, functions $\Phi, h$ and viscosity solutions $v$ and $\widehat{v}$

$$
\begin{aligned}
0= & v_{\mathbf{n}_{0}}(T, 0)-\widehat{v}_{\mathbf{n}_{0}}(T, 0)+2 h\left(\varepsilon-L_{0} T_{0}\right), \\
& \left(\text { since } v_{\mathbf{n}_{0}}(T, 0)=\widehat{v}_{\mathbf{n}_{0}}(T, 0)=h\left(\varepsilon-L_{0} T_{0}\right)=0\right) \\
\leq & v_{\mathbf{n}_{0}}\left(t_{0}, \zeta_{1}^{0}\right)-\widehat{v}_{\mathbf{n}_{0}}\left(s_{0}, \zeta_{2}^{0}\right)-\frac{1}{\alpha}\left\|\zeta_{1}^{0}-\zeta_{2}^{0}\right\|^{2}-\frac{1}{\beta}\left[t_{0}-s_{0}\right]^{2} \\
& \quad+h\left(\left\langle\zeta_{1}^{0}\right\rangle_{\varepsilon}-L_{0}\left[t_{0}-T+T_{0}\right]\right)+h\left(\left\langle\zeta_{2}^{0}\right\rangle_{\varepsilon}-L_{0}\left[s_{0}-T+T_{0}\right]\right)+\gamma\left[t_{0}+s_{0}\right]-2 \gamma T
\end{aligned}
$$

$$
<K+h\left(\left\langle\zeta_{1}^{0}\right\rangle_{\varepsilon}-L_{0}\left[t_{0}-T+T_{0}\right]\right)+h\left(\left\langle\zeta_{2}^{0}\right\rangle_{\varepsilon}-L_{0}\left[s_{0}-T+T_{0}\right]\right)+\gamma\left[t_{0}+s_{0}\right]-2 \gamma T
$$

By (4.13) and (4.15), we have $t_{0}+s_{0}>2 T$, which is a contradiction since $t_{0}, s_{0} \leq T$.

Proposition 4.1. (Following M. Huang et al [8]) For any $\alpha, \beta, \gamma, \varepsilon, \delta>0$, such that $\varepsilon+\delta<L_{0} T_{0}$ and $\alpha, \beta<\epsilon_{0}$, for some $\epsilon_{0}>0, \Phi$ achieves its maximum point over the compact set $\overline{\mathcal{N} \times \mathbb{N} \times \mathbb{N}}$ at $\left(\mathbf{n}_{0}, t_{0}, \zeta_{1}^{0}, s_{0}, \zeta_{2}^{0}\right) \in \mathcal{N} \times \mathbb{N} \times \mathbb{N}$.

Proof. Suppose that $\Phi$ achieves its maximum point over the compact set $\overline{\mathcal{N} \times \mathbb{N} \times \mathbb{N}}$ at $\left(\mathbf{n}_{0}, t_{0}, \zeta_{1}^{0}, s_{0}, \zeta_{2}^{0}\right)$, then we have

$$
\Phi_{\mathbf{n}_{0}}\left(t_{0}, \zeta_{1}^{0}, t_{0}, \zeta_{1}^{0}\right)+\Phi_{\mathbf{n}_{0}}\left(s_{0}, \zeta_{2}^{0}, s_{0}, \zeta_{2}^{0}\right) \leq 2 \Phi_{\mathbf{n}_{0}}\left(t_{0}, \zeta_{1}^{0}, s_{0}, \zeta_{2}^{0}\right),
$$

which implies that, by (4.9)

$$
\begin{aligned}
& \quad v_{\mathbf{n}_{0}}\left(t_{0}, \zeta_{1}^{0}\right)-\widehat{v}_{\mathbf{n}_{0}}\left(t_{0}, \zeta_{1}^{0}\right)+2 h\left(\left\langle\zeta_{1}^{0}\right\rangle_{\varepsilon}-L_{0}\left[t_{0}-T+T_{0}\right]\right) \\
& \quad+v_{\mathbf{n}_{0}}\left(s_{0}, \zeta_{2}^{0}\right)-\widehat{v}_{\mathbf{n}_{0}}\left(s_{0}, \zeta_{2}^{0}\right)+2 h\left(\left\langle\zeta_{2}^{0}\right\rangle_{\varepsilon}-L_{0}\left[s_{0}-T+T_{0}\right]\right)+2 \gamma\left[t_{0}+s_{0}\right]-4 \gamma T, \\
& \leq 2 v_{\mathbf{n}_{0}}\left(t_{0}, \zeta_{1}^{0}\right)-2 \widehat{v}_{\mathbf{n}_{0}}\left(s_{0}, \zeta_{2}^{0}\right)-\frac{2}{\alpha}\left\|\zeta_{1}^{0}-\zeta_{2}^{0}\right\|^{2}-\frac{2}{\beta}\left[t_{0}-s_{0}\right]^{2} \\
& \quad+2 h\left(\left\langle\zeta_{1}^{0}\right\rangle_{\varepsilon}-L_{0}\left[t_{0}-T+T_{0}\right]\right)+2 h\left(\left\langle\zeta_{2}^{0}\right\rangle_{\varepsilon}-L_{0}\left[s_{0}-T+T_{0}\right]\right)+2 \gamma\left[t_{0}+s_{0}\right]-4 \gamma T,
\end{aligned}
$$

which yields the following inequality

$$
\begin{aligned}
\frac{2}{\alpha}\left\|\zeta_{1}^{0}-\zeta_{2}^{0}\right\|^{2}+\frac{2}{\beta}\left[t_{0}-s_{0}\right]^{2} & \leq v_{\mathbf{n}_{0}}\left(t_{0}, \zeta_{1}^{0}\right)-v_{\mathbf{n}_{0}}\left(s_{0}, \zeta_{2}^{0}\right)+\widehat{v}_{\mathbf{n}_{0}}\left(t_{0}, \zeta_{1}^{0}\right)-\widehat{v}_{\mathbf{n}_{0}}\left(s_{0}, \zeta_{2}^{0}\right) \\
& \leq 2 \eta\left(\left|t_{0}-s_{0}\right|+\left\|\zeta_{1}^{0}-\zeta_{2}^{0}\right\|\right)
\end{aligned}
$$


where $\eta$ is specified as the following:

$$
\eta(\mu) \triangleq \frac{1}{2} \sup _{\substack{|t-s|+|| \zeta_{1}-\zeta_{2}|| \leq \mu \\\left(t, \zeta_{1}, s, \zeta_{2}\right) \in \mathbb{N}\left(\mathbf{n}_{0}, T_{0}\right) \times \mathbb{N}\left(\mathbf{n}_{0}, T_{0}\right)}}\left\{\left|v_{\mathbf{n}_{0}}\left(t, \zeta_{1}\right)-v_{\mathbf{n}_{0}}\left(s, \zeta_{2}\right)\right|+\left|\widehat{v}_{\mathbf{n}_{0}}\left(t, \zeta_{1}\right)-\widehat{v}_{\mathbf{n}_{0}}\left(s, \zeta_{2}\right)\right|\right\}
$$

By the continuity property of viscosity solutions $v_{\mathbf{n}_{0}}$ and $\widehat{v}_{\mathbf{n}_{0}}$ and boundedness of the set $\mathbb{N}\left(\mathbf{n}_{0}, T_{0}\right)$, we have

$$
\eta^{\star} \triangleq \sup _{\nu>0} \eta(\nu)<\infty \quad \text { and } \quad \lim _{\nu \rightarrow 0} \eta(\nu)=0
$$

Also by (4.17) and (4.19), we obtain that

$$
\begin{aligned}
|| \zeta_{1}^{0}-\zeta_{2}^{0}|| & \leq \sqrt{\alpha \eta^{\star}} \\
\left|t_{0}-s_{0}\right| & \leq \sqrt{\beta \eta^{\star}}
\end{aligned}
$$

Hence, by (4.18), (4.17), (4.20) and (4.21), the following holds

$$
\frac{1}{\alpha}\left\|\zeta_{1}^{0}-\zeta_{2}^{0}\right\|^{2}+\frac{1}{\beta}\left[t_{0}-s_{0}\right]^{2} \leq \eta\left(\sqrt{\alpha \eta^{\star}}+\sqrt{\beta \eta^{\star}}\right)
$$

We define $\mathbb{N}_{\varepsilon, \delta} \equiv \mathbb{N}_{\varepsilon, \delta}\left(\mathbf{n}_{0}, T_{0}\right)$ as

$$
\mathbb{N}_{\varepsilon, \delta} \triangleq\left\{(t, \zeta) \in \mathbb{N}\left(\mathbf{n}_{0}, T_{0}\right) ;\langle\zeta\rangle_{\varepsilon} \leq L_{0}\left[t-T+T_{0}\right]-\delta\right\} .
$$

There exist small enough positive values of $\varepsilon, \delta, \gamma$, such that

$$
\sup _{(t, \zeta) \in \mathbb{N}_{\varepsilon, \delta}} \Phi_{\mathbf{n}_{0}}(t, \zeta, t, \zeta)=\sup _{(t, \zeta) \in \mathbb{N}_{\varepsilon, \delta}}\left\{v_{\mathbf{n}_{0}}(t, \zeta)-\widehat{v}_{\mathbf{n}_{0}}(t, \zeta)+2 \gamma[t-T]\right\} \geq \frac{\bar{\gamma}}{2}>0
$$

The Proof of (4.24).

The value of $\mathbf{n}_{0}$ is dependent upon the value of $\alpha, \beta, \gamma, \varepsilon$ and $\delta$; and $\left(\mathbf{n}_{0}, t_{0}, \zeta_{1}^{0}, s_{0}\right.$, $\left.\zeta_{2}^{0}\right)=\operatorname{argmax}_{\left(\mathbf{n}, t, \zeta_{1}, s, \zeta_{2}\right) \in \overline{\mathcal{N} \times \mathbb{N} \times \mathbb{N}}}\left\{\Phi_{\mathbf{n}}\left(t, \zeta_{1}, s, \zeta_{2}\right)\right\}$, with the function $\Phi_{\mathbf{n}}\left(t, \zeta_{1}, s, \zeta_{2}\right)$ specified in (4.9), hence

$$
\Phi_{\mathbf{n}_{0}}(t, \zeta, t, \zeta)=v_{\mathbf{n}_{0}}(t, \zeta)-\widehat{v}_{\mathbf{n}_{0}}(t, \zeta)+2 \gamma[t-T]
$$

And the strictly positive real number $\bar{\gamma}$, is specified in (4.5), i.e.

$$
\max _{\mathbf{n} \in \mathcal{N}}\left\{\sup _{(s, \zeta) \in \mathbb{N}\left(\mathbf{n}, T_{0}\right)}\left\{v_{\mathbf{n}}(s, \zeta)-\widehat{v}_{\mathbf{n}}(s, \zeta)\right\}\right\} \geq \bar{\gamma}>0 .
$$

Completion of the Proof of (4.24).

Also, by the specification of the function $\Phi$, we observe that

$$
\begin{aligned}
\sup _{(t, \zeta) \in \mathbb{N}_{\varepsilon, \delta}} \Phi_{\mathbf{n}_{0}}(t, \zeta, t, \zeta) & \leq \sup _{\left(t, \zeta_{1}, s, \zeta_{2}\right) \in \mathbb{N} \times \mathbb{N}} \Phi_{\mathbf{n}_{0}}\left(t, \zeta_{1}, s, \zeta_{2}\right) \\
& =\Phi_{\mathbf{n}_{0}}\left(t_{0}, \zeta_{1}^{0}, s_{0}, \zeta_{2}^{0}\right) \leq v_{\mathbf{n}_{0}}\left(t_{0}, \zeta_{1}^{0}\right)-\widehat{v}_{\mathbf{n}_{0}}\left(s_{0}, \zeta_{2}^{0}\right)
\end{aligned}
$$


Next we show that there exists a strictly positive real number $\epsilon_{0}$, such that for any $0<\alpha, \beta<\epsilon_{0}$,

$$
\left(\mathbf{n}_{0}, t_{0}, \zeta_{1}^{0}, s_{0}, \zeta_{2}^{0}\right) \in \mathcal{N} \times \mathbb{N} \times \mathbb{N}
$$

Suppose that there does not exist such an $\epsilon_{0}$. In other words, suppose that, for any $\epsilon>$ 0 , there exist some $\alpha_{\epsilon}, \beta_{\epsilon}, 0<\alpha_{\epsilon}, \beta_{\epsilon}<\epsilon$, such that $\left(\mathbf{n}_{0}, t_{0}, \zeta_{1}^{0}, s_{0}, \zeta_{2}^{0}\right) \in \partial(\mathcal{N} \times \mathbb{N} \times \mathbb{N})$ the boundary set of $\mathcal{N} \times \mathbb{N} \times \mathbb{N}$. Then by Lemma 4.2 and (4.4), we obtain that

$$
t_{0}=T \text { or } s_{0}=T \text { or } t_{0}=T-T_{0} \text { or } s_{0}=T-T_{0}
$$

We can exclude the two latter cases $t_{0}=T-T_{0}$ and $s_{0}=T-T_{0}$, since, if $t_{0}=T-T_{0}$ or $s_{0}=T-T_{0}$, by the specification of $\mathbb{N}$, we obtain that $\left\|\zeta_{1}^{0}\right\|<L_{0}\left[t_{0}-T+T_{0}\right]=0$ or $\left\|\zeta_{2}^{0}\right\|<L_{0}\left[s_{0}-T+T_{0}\right]=0$, which is a contradiction.

In case of $t_{0}=T$ : Then, by the specification of a viscosity solution, we have

$$
v_{\mathbf{n}_{0}}\left(t_{0}, \zeta_{1}^{0}\right)=v_{\mathbf{n}_{0}}\left(T, \zeta_{1}^{0}\right)=0, \quad \text { for any } \zeta_{1}^{0} \in \mathbb{R}_{+}^{d\left(\mathbf{n}_{0}\right)}
$$

By (4.21), i.e. $\left|t_{0}-s_{0}\right|<\sqrt{\beta_{\epsilon} \eta^{\star}}$, and the continuity property of the viscosity solution $\widehat{v}_{\mathbf{n}_{0}}$, and $\widehat{v}_{\mathbf{n}_{0}}\left(T, \zeta_{2}^{0}\right)=0$, for all $\zeta_{2}^{0} \in \mathbb{R}_{+}^{d\left(\mathbf{n}_{0}\right)}$, there exists an $\epsilon^{\prime}>0$, such that

$$
\left|v_{\mathbf{n}_{0}}\left(t_{0}, \zeta_{1}^{0}\right)-\widehat{v}_{\mathbf{n}_{0}}\left(s_{0}, \zeta_{2}^{0}\right)\right|=\left|\widehat{v}_{\mathbf{n}_{0}}\left(s_{0}, \zeta_{2}^{0}\right)\right| \leq \frac{\bar{\gamma}}{4}
$$

Hence, by (4.24), (4.25), (4.27) and (4.28), we obtain that

$$
0<\frac{\bar{\gamma}}{2} \leq \sup _{(t, \zeta) \in \mathbb{N}_{\varepsilon, \delta}} \Phi_{\mathbf{n}_{0}}(t, \zeta, t, \zeta) \leq v_{\mathbf{n}_{0}}\left(t_{0}, \zeta_{1}^{0}\right)-\widehat{v}_{\mathbf{n}_{0}}\left(s_{0}, \zeta_{2}^{0}\right) \leq \frac{\bar{\gamma}}{4}
$$

which is a contradiction. Similarly, consider that $s_{0}=T$, one may also obtain a contradiction. Thus (4.26) holds. Hence we proved that, for any $\alpha, \beta, \gamma, \varepsilon, \delta>0$, such that $\varepsilon+\delta<L_{0} T_{0}$ and $\alpha, \beta<\epsilon_{0}$, for some $\epsilon_{0}>0$, $\Phi$ attains its maximum point over the compact set $\overline{\mathcal{N} \times \mathbb{N} \times \mathbb{N}}$ at $\left(\mathbf{n}_{0}, t_{0}, \zeta_{1}^{0}, s_{0}, \zeta_{2}^{0}\right) \in \mathcal{N} \times \mathbb{N} \times \mathbb{N}$.

Lemma 4.3. For any $e \in E_{\mathbf{n}_{0}}$ and $\zeta_{1}^{0}, \zeta_{2}^{0} \in \mathbb{R}_{+}^{d\left(\mathbf{n}_{0}\right)}$, subject to any $u \in U\left(\mathbf{n}_{0}, e\right)$, we have

$$
v_{\mathbf{n}_{0}}\left(t_{0}, \zeta_{1}^{0}\right)-\widehat{v}_{\mathbf{n}_{0}}\left(s_{0}, \zeta_{2}^{0}\right) \geq v_{\mathbf{n}_{0}+u}\left(t_{0}, \zeta_{1}^{0} \circ u\right)-\widehat{v}_{\mathbf{n}_{0}+u}\left(s_{0}, \zeta_{2}^{0} \circ u\right),
$$

Proof. By Lemma 4.1, we have $\left\|\zeta_{1}^{0} \circ u\right\| \leq\left\|\zeta_{1}^{0}\right\|$ and $\left\|\zeta_{2}^{0} \circ u\right\| \leq\left\|\zeta_{2}^{0}\right\|$, which implies that $\left(\mathbf{n}_{0}+u, t_{0}, \zeta_{1}^{0} \circ u, s_{0}, \zeta_{2}^{0} \circ u\right) \in \overline{\mathcal{N} \times \mathbb{N} \times \mathbb{N}}$, then we have

$$
\Phi_{\mathbf{n}_{0}}\left(t_{0}, \zeta_{1}^{0}, s_{0}, \zeta_{2}^{0}\right) \geq \Phi_{\mathbf{n}_{0}+u}\left(t_{0}, \zeta_{1}^{0} \circ u, s_{0}, \zeta_{2}^{0} \circ u\right)
$$

since $\Phi$ attains its maximum point over $\overline{\mathcal{N} \times \mathbb{N} \times \mathbb{N}}$ at $\left(\mathbf{n}_{0}, t_{0}, \zeta_{1}^{0}, s_{0}, \zeta_{2}^{0}\right)$. 
By the inequality (4.31) and the definition of $\Phi$ given in (4.9), we obtain that

$$
\begin{aligned}
& v_{\mathbf{n}_{0}}\left(t_{0}, \zeta_{1}^{0}\right)-\widehat{v}_{\mathbf{n}_{0}}\left(s_{0}, \zeta_{2}^{0}\right)-\frac{1}{\alpha}\left\|\zeta_{1}^{0}-\zeta_{2}^{0}\right\|^{2} \\
& \quad+h\left(\left\langle\zeta_{1}^{0}\right\rangle_{\varepsilon}-L_{0}\left[t_{0}-T+T_{0}\right]\right)+h\left(\left\langle\zeta_{2}^{0}\right\rangle_{\varepsilon}-L_{0}\left[s_{0}-T+T_{0}\right]\right) \\
& \geq v_{\mathbf{n}_{0}+u}\left(t_{0}, \zeta_{1}^{0} \circ u\right)-\widehat{v}_{\mathbf{n}_{0}+u}\left(s_{0}, \zeta_{2}^{0} \circ u\right)-\frac{1}{\alpha}\left\|\zeta_{1}^{0} \circ u-\zeta_{2}^{0} \circ u\right\|^{2} \\
& \quad+h\left(\left\langle\zeta_{1}^{0} \circ u\right\rangle_{\varepsilon}-L_{0}\left[t_{0}-T+T_{0}\right]\right)+h\left(\left\langle\zeta_{2}^{0} \circ u\right\rangle_{\varepsilon}-L_{0}\left[s_{0}-T+T_{0}\right]\right)
\end{aligned}
$$

By Lemma 4.1, we have

$$
\left\|\zeta_{1}^{0} \circ u-\zeta_{2}^{0} \circ u\right\| \leq\left\|\zeta_{1}^{0}-\zeta_{2}^{0}\right\|
$$

Also, for any $\zeta \in \mathbb{R}_{+}^{d(\mathbf{n})}$, by Lemma 4.1 , we have $\|\zeta \circ u\| \leq\|\zeta\|$, then

$$
\langle\zeta \circ u\rangle_{\varepsilon}=\sqrt{\|\zeta \circ u\|^{2}+\varepsilon^{2}} \leq \sqrt{\|\zeta\|^{2}+\varepsilon^{2}}=\langle\zeta\rangle_{\varepsilon}
$$

By (4.34) and non-increasing property of the function $h: \mathbb{R} \rightarrow \mathbb{R}$, we have

$$
\begin{aligned}
& h\left(\left\langle\zeta_{1}^{0} \circ u\right\rangle_{\varepsilon}-L_{0}\left[t_{0}-T+T_{0}\right]\right) \geq h\left(\left\langle\zeta_{1}^{0}\right\rangle_{\varepsilon}-L_{0}\left[t_{0}-T+T_{0}\right]\right) \\
& h\left(\left\langle\zeta_{2}^{0} \circ u\right\rangle_{\varepsilon}-L_{0}\left[s_{0}-T+T_{0}\right]\right) \geq h\left(\left\langle\zeta_{2}^{0}\right\rangle_{\varepsilon}-L_{0}\left[s_{0}-T+T_{0}\right]\right)
\end{aligned}
$$

So, by the inequality of (4.32), we have

$$
\begin{aligned}
& \quad\left(v_{\mathbf{n}_{0}}\left(t_{0}, \zeta_{1}^{0}\right)-\widehat{v}_{\mathbf{n}_{0}}\left(s_{0}, \zeta_{2}^{0}\right)\right)-\left(v_{\mathbf{n}_{0}+u}\left(t_{0}, \zeta_{1}^{0} \circ u\right)-\widehat{v}_{\mathbf{n}_{0}+u}\left(s_{0}, \zeta_{2}^{0} \circ u\right)\right) \\
& \geq \frac{1}{\alpha}\left(\left\|\zeta_{1}^{0}-\zeta_{2}^{0}\right\|^{2}-\left\|\zeta_{1}^{0} \circ u-\zeta_{2}^{0} \circ u\right\|^{2}\right) \\
& \quad+\left(h\left(\left\langle\zeta_{1}^{0} \circ u\right\rangle_{\varepsilon}-L_{0}\left[t_{0}-T+T_{0}\right]\right)-h\left(\left\langle\zeta_{1}^{0}\right\rangle_{\varepsilon}-L_{0}\left[t_{0}-T+T_{0}\right]\right)\right) \\
& \quad+\left(h\left(\left\langle\zeta_{2}^{0} \circ u\right\rangle_{\varepsilon}-L_{0}\left[s_{0}-T+T_{0}\right]\right)-h\left(\left\langle\zeta_{2}^{0}\right\rangle_{\varepsilon}-L_{0}\left[s_{0}-T+T_{0}\right]\right)\right) \\
& \quad
\end{aligned}
$$

the last inequality holds by (4.33), (4.35) and(4.36), which is the inequality (4.30), the conclusion.

(Step 2) Viscosity subsolutions and supersolutions of the hybrid HJB equation (2.17).

TheOREm 4.1. Under assumptions (S1)-(S5), the hybrid HJB equation (2.17) has at most one viscosity solution in $C\left([0, T] \times \mathbb{R}_{+}^{d(\mathbf{n})}\right)$.

Proof. (i) Define a function $\phi_{\mathbf{n}_{0}}: \mathbb{N}\left(\mathbf{n}_{0}, T_{0}\right) \rightarrow \mathbb{R}$ as the following:

$$
\phi_{\mathbf{n}_{0}}\left(t, \zeta_{1}\right) \triangleq \widehat{v}_{\mathbf{n}_{0}}\left(s_{0}, \zeta_{2}^{0}\right)+\frac{1}{\alpha}\left\|\zeta_{1}-\zeta_{2}^{0}\right\|^{2}+\frac{1}{\beta}\left[t-s_{0}\right]^{2}
$$

Then for $0<\alpha, \beta<\epsilon_{0}$, by the definition of $\left(\mathbf{n}_{0}, t_{0}, \zeta_{1}^{0}, s_{0}, \zeta_{2}^{0}\right)$ and Proposition 4.1, we have $v_{\mathbf{n}_{0}}\left(t, \zeta_{1}\right)-\phi_{\mathbf{n}_{0}}\left(t, \zeta_{1}\right)$ attains the global maximum value at $\left(t_{0}, \zeta_{1}^{0}\right) \in \mathbb{N}\left(\mathbf{n}_{0}, T_{0}\right)$. 
Since by the hypothesis $v$ is a viscosity subsolution of (2.17), we obtain that

$$
\begin{aligned}
0 & \left.\left(\left(\frac{\partial}{\partial t}+\sum_{i=1}^{d\left(\mathbf{n}_{0}\right)} \frac{\partial}{\partial \zeta_{1}^{i}}\right) \phi_{\mathbf{n}_{0}}\left(t, \zeta_{1}\right)\right)\right|_{\left(t_{0}, \zeta_{1}^{0}\right)}+g_{\mathbf{n}_{0}}\left(t_{0}, \zeta_{1}^{0}\right) \\
& +\sum_{e \in E_{\mathbf{n}_{0}}} \lambda^{e}\left(\zeta_{1}^{0}\right)\left(\min _{u \in U\left(\mathbf{n}_{0}, \zeta_{1}^{0}, e\right)} v_{\mathbf{n}_{0}+u}\left(t_{0}, \zeta_{1}^{0} \circ u\right)-v_{\mathbf{n}_{0}}\left(t_{0}, \zeta_{1}^{0}\right)\right) \\
= & \frac{2}{\beta}\left[t_{0}-s_{0}\right]+\frac{2}{\alpha}\left(\sum_{i=1}^{d\left(\mathbf{n}_{0}\right)}\left[\zeta_{1}^{0, i}-\zeta_{2}^{0, i}\right]\right)-\gamma \\
- & \left.\left(-L_{0}+\frac{\sum_{i=1}^{d\left(\mathbf{n}_{0}\right)} \zeta_{1}^{0, i}}{\left\langle\zeta_{1}^{0}\right\rangle_{\varepsilon}}\right) \frac{d h(y)}{d y}\right|_{A}+g_{\mathbf{n}_{0}}\left(t_{0}, \zeta_{1}^{0}\right) \\
+ & \sum_{e \in E_{\mathbf{n}_{0}}} \lambda^{e}\left(\zeta_{1}^{0}\right)\left(\min _{u \in U\left(\mathbf{n}_{0}, \zeta_{1}^{0}, e\right)} v_{\mathbf{n}_{0}+u}\left(t_{0}, \zeta_{1}^{0} \circ u\right)-v_{\mathbf{n}_{0}}\left(t_{0}, \zeta_{1}^{0}\right)\right)
\end{aligned}
$$

where $A \triangleq\left\langle\zeta_{1}^{0}\right\rangle_{\varepsilon}-L_{0}\left[t_{0}-T+T_{0}\right]$.

(ii) Define a function $\psi_{\mathbf{n}_{0}}: \mathbb{N}\left(\mathbf{n}_{0}, T_{0}\right) \rightarrow \mathbb{R}$ as the following:

$$
\begin{aligned}
& \psi_{\mathbf{n}_{0}}\left(s, \zeta_{2}\right) \triangleq v_{\mathbf{n}_{0}}\left(t_{0}, \zeta_{1}^{0}\right)-\frac{1}{\alpha}\left\|\zeta_{1}^{0}-\zeta_{2}\right\|^{2}-\frac{1}{\beta}\left[t_{0}-s\right]^{2} \\
& (4.39)+h\left(\left\langle\zeta_{1}^{0}\right\rangle_{\varepsilon}-L_{0}\left[t_{0}-T+T_{0}\right]\right)+h\left(\left\langle\zeta_{2}\right\rangle_{\varepsilon}-L_{0}\left[s-T+T_{0}\right]\right)+\gamma\left[t_{0}+s\right]-2 \gamma T
\end{aligned}
$$

Then for $0<\alpha, \beta<\epsilon_{0}$, by Proposition (4.1), we have $\widehat{v}_{\mathbf{n}_{0}}\left(s, \zeta_{2}\right)-\psi_{\mathbf{n}_{0}}\left(s, \zeta_{2}\right)$ attains the global minimum at $\left(s_{0}, \zeta_{2}^{0}\right) \in \mathbb{N}\left(\mathbf{n}_{0}, T_{0}\right)$.

Since by the assumption, $\widehat{v}$ is a viscosity supersolution of (2.17), we obtain that

$$
\begin{aligned}
0 \geq & \left.\left(\left(\frac{\partial}{\partial s}+\sum_{i=1}^{d\left(\mathbf{n}_{0}\right)} \frac{\partial}{\partial \zeta_{2}^{i}}\right) \psi_{\mathbf{n}_{0}}\left(s, \zeta_{2}\right)\right)\right|_{\left(s_{0}, \zeta_{2}^{0}\right)}+g_{\mathbf{n}_{0}}\left(s_{0}, \zeta_{2}^{0}\right) \\
& +\sum_{e \in E_{\mathbf{n}_{0}}} \lambda^{e}\left(\zeta_{2}^{0}\right)\left(\min _{u \in U\left(\mathbf{n}_{0}, \zeta_{2}^{0}, e\right)} \widehat{\mathbf{n}}_{\mathbf{n}_{0}+u}\left(s_{0}, \zeta_{2}^{0} \circ u\right)-\widehat{v}_{\mathbf{n}_{0}}\left(s_{0}, \zeta_{2}^{0}\right)\right) \\
= & \frac{2}{\beta}\left[t_{0}-s_{0}\right]+\frac{2}{\alpha}\left(\sum_{i=1}^{d\left(\mathbf{n}_{0}\right)}\left[\zeta_{1}^{0, i}-\zeta_{2}^{0, i}\right]\right)+\gamma \\
+ & \left.\left(-L_{0}+\frac{\sum_{i=1}^{d\left(\mathbf{n}_{0}\right)} \zeta_{2}^{0, i}}{\left\langle\zeta_{2}^{0}\right\rangle_{\varepsilon}}\right) \frac{d h(y)}{d y}\right|_{B}+g_{\mathbf{n}_{0}}\left(s_{0}, \zeta_{2}^{0}\right) \\
& +\sum_{e \in E_{\mathbf{n}_{0}}} \lambda^{e}\left(\zeta_{2}^{0}\right)\left(\min _{u \in U\left(\mathbf{n}_{0}, \zeta_{2}^{0}, e\right)} \widehat{v}_{\mathbf{n}_{0}+u}\left(s_{0}, \zeta_{2}^{0} \circ u\right)-\widehat{v}_{\mathbf{n}_{0}}\left(s_{0}, \zeta_{2}^{0}\right)\right)
\end{aligned}
$$

where $\quad B \triangleq\left\langle\zeta_{2}^{0}\right\rangle_{\varepsilon}-L_{0}\left[s_{0}-T+T_{0}\right]$ 
Hence by (4.38) and (4.40), we obtain that

$$
\begin{aligned}
2 \gamma \leq L_{0}\left(\left.\frac{d h(y)}{d y}\right|_{A}+\left.\frac{d h(y)}{d y}\right|_{B}\right)-\left(\left.\frac{\sum_{i=1}^{d\left(\mathbf{n}_{0}\right)} \zeta_{1}^{0, i}}{\left\langle\zeta_{1}^{0}\right\rangle_{\varepsilon}} \frac{d h(y)}{d y}\right|_{A}+\left.\frac{\sum_{i=1}^{d\left(\mathbf{n}_{0}\right)} \zeta_{2}^{0, i}}{\left\langle\zeta_{2}^{0}\right\rangle_{\varepsilon}} \frac{d h(y)}{d y}\right|_{B}\right) & \triangleq(1) \\
+\left(g_{\mathbf{n}_{0}}\left(t_{0}, \zeta_{1}^{0}\right)-g_{\mathbf{n}_{0}}\left(s_{0}, \zeta_{2}^{0}\right)\right) & \triangleq(2) \\
+\left(\sum_{e \in E_{\mathbf{n}_{0}}} \lambda^{e}\left(\zeta_{1}^{0}\right)\left(\min _{u \in U\left(\mathbf{n}_{0}, \zeta_{1}^{0}, e\right)} v_{\mathbf{n}_{0}+u}\left(t_{0}, \zeta_{1}^{0} \circ u\right)-v_{\mathbf{n}_{0}}\left(t_{0}, \zeta_{1}^{0}\right)\right)\right. & \\
\left.\quad-\sum_{e \in E_{\mathbf{n}_{0}}} \lambda^{e}\left(\zeta_{2}^{0}\right)\left(\min _{u \in U\left(\mathbf{n}_{0}, \zeta_{2}^{0}, e\right)} \widehat{v}_{\mathbf{n}_{0}+u}\left(s_{0}, \zeta_{2}^{0} \circ u\right)-\widehat{v}_{\mathbf{n}_{0}}\left(s_{0}, \zeta_{2}^{0}\right)\right)\right) & \triangleq(3) \\
(4.41) & \\
\equiv(1)+(2)+(3) . &
\end{aligned}
$$

For any $\mathbf{n}_{0} \in \mathcal{N}, \zeta \in \mathbb{R}_{+}^{d\left(\mathbf{n}_{0}\right)}$ and $\varepsilon>0$, we observe that

$$
\frac{\sum_{i=1}^{d\left(\mathbf{n}_{0}\right)} \zeta^{i}}{\langle\zeta\rangle_{\varepsilon}}<\frac{\sum_{i=1}^{d\left(\mathbf{n}_{0}\right)} \zeta^{i}}{\|\zeta\|} \leq \sqrt{d\left(\mathbf{n}_{0}\right)}<\sqrt{d\left(\mathbf{n}_{0}\right)+1}<L_{0} .
$$

Then by the inequality (4.42) and $\frac{d h(y)}{d y} \leq 0$ by (4.8), we have

$$
(1)=\left.\left(L_{0}-\frac{\sum_{i=1}^{d\left(\mathbf{n}_{0}\right)} \zeta_{1}^{0, i}}{\left\langle\zeta_{1}^{0}\right\rangle_{\varepsilon}}\right) \frac{d h(y)}{d y}\right|_{A}+\left.\left(L_{0}-\frac{\sum_{i=1}^{d\left(\mathbf{n}_{0}\right)} \zeta_{2}^{0, i}}{\left\langle\zeta_{2}^{0}\right\rangle_{\varepsilon}}\right) \frac{d h(y)}{d y}\right|_{B} \leq 0
$$

By assumption (S5), we obtain that

$$
\begin{array}{rlr}
(2) & \leq\left|g_{\mathbf{n}_{0}}\left(t_{0}, \zeta_{1}^{0}\right)-g_{\mathbf{n}_{0}}\left(s_{0}, \zeta_{2}^{0}\right)\right| \\
& \leq\left|g_{\mathbf{n}_{0}}\left(t_{0}, \zeta_{1}^{0}\right)-g_{\mathbf{n}_{0}}\left(s_{0}, \zeta_{1}^{0}\right)\right|+\left|g_{\mathbf{n}_{0}}\left(s_{0}, \zeta_{1}^{0}\right)-g_{\mathbf{n}_{0}}\left(s_{0}, \zeta_{2}^{0}\right)\right| \\
& \leq L\left|t_{0}-s_{0}\right|+L|| \zeta_{1}^{0}-\zeta_{2}^{0}|| \\
& \leq L\left(\sqrt{\alpha \eta^{\star}}+\sqrt{\beta \eta^{\star}}\right), \quad \text { by }(4.20) \text { and }(4.21)
\end{array}
$$

And carrying out a pairwise difference procedure for (3), we obtain that

$$
\begin{aligned}
& (3)=\left\{\sum_{e \in E_{\mathbf{n}_{0}}} \lambda^{e}\left(\zeta_{1}^{0}\right)\left(\min _{u \in U\left(\mathbf{n}_{0}, \zeta_{1}^{0}, e\right)} v_{\mathbf{n}_{0}+u}\left(t_{0}, \zeta_{1}^{0} \circ u\right)-v_{\mathbf{n}_{0}}\left(t_{0}, \zeta_{1}^{0}\right)\right)\right. \\
& \left.\quad-\sum_{e \in E_{\mathbf{n}_{0}}} \lambda^{e}\left(\zeta_{1}^{0}\right)\left(\min _{u \in U\left(\mathbf{n}_{0}, \zeta_{1}^{0}, e\right)} \widehat{v}_{\mathbf{n}_{0}+u}\left(s_{0}, \zeta_{2}^{0} \circ u\right)-\widehat{v}_{\mathbf{n}_{0}}\left(s_{0}, \zeta_{2}^{0}\right)\right)\right\} \triangleq(4) \\
& +\left\{\sum_{e \in E_{\mathbf{n}_{0}}} \lambda^{e}\left(\zeta_{1}^{0}\right)\left(\min _{u \in U\left(\mathbf{n}_{0}, \zeta_{1}^{0}, e\right)} \widehat{v}_{\mathbf{n}_{0}+u}\left(s_{0}, \zeta_{2}^{0} \circ u\right)-\widehat{v}_{\mathbf{n}_{0}}\left(s_{0}, \zeta_{2}^{0}\right)\right)\right. \\
& \left.(4.45) \quad-\sum_{e \in E_{\mathbf{n}_{0}}} \lambda^{e}\left(\zeta_{2}^{0}\right)\left(\min _{u \in U\left(\mathbf{n}_{0}, \zeta_{2}^{0}, e\right)} \widehat{v}_{\mathbf{n}_{0}+u}\left(s_{0}, \zeta_{2}^{0} \circ u\right)-\widehat{v}_{\mathbf{n}_{0}}\left(s_{0}, \zeta_{2}^{0}\right)\right)\right\} \triangleq(5),
\end{aligned}
$$


with (4) and (5) satisfy the follows respectively.

$$
\begin{aligned}
(4)= & -\sum_{e \in E_{\mathbf{n}_{0}}} \lambda^{e}\left(\zeta_{1}^{0}\right)\left(v_{\mathbf{n}_{0}}\left(t_{0}, \zeta_{1}^{0}\right)-\widehat{v}_{\mathbf{n}_{0}}\left(s_{0}, \zeta_{2}^{0}\right)\right) \\
& +\sum_{e \in E_{\mathbf{n}_{0}}} \lambda^{e}\left(\zeta_{1}^{0}\right)\left(\min _{u \in U\left(\mathbf{n}_{0}, e\right)} v_{\mathbf{n}_{0}+u}\left(t_{0}, \zeta_{1}^{0} \circ u\right)-\min _{u \in U\left(\mathbf{n}_{0}, e\right)} \widehat{v}_{\mathbf{n}_{0}+u}\left(s_{0}, \zeta_{2}^{0} \circ u\right)\right) \\
\leq & -\sum_{e \in E_{\mathbf{n}_{0}}} \lambda^{e}\left(\zeta_{1}^{0}\right)\left(v_{\mathbf{n}_{0}}\left(t_{0}, \zeta_{1}^{0}\right)-\widehat{v}_{\mathbf{n}_{0}}\left(s_{0}, \zeta_{2}^{0}\right)\right) \\
(4.46) \quad & +\sum_{e \in E_{\mathbf{n}_{0}}} \lambda^{e}\left(\zeta_{1}^{0}\right) \max _{u \in U\left(\mathbf{n}_{0}, e\right)}\left\{v_{\mathbf{n}_{0}+u}\left(t_{0}, \zeta_{1}^{0} \circ u\right)-\widehat{v}_{\mathbf{n}_{0}+u}\left(s_{0}, \zeta_{2}^{0} \circ u\right)\right\}, \\
\leq & 0, \quad \text { by Lemma } 4.3 .
\end{aligned}
$$

Remark: in the above inequality analysis we applied that $\min _{y \in Y} f_{1}(y)-\min _{y \in Y} f_{2}(y)$ $\leq \max _{y \in Y}\left\{f_{1}(y)-f_{2}(y)\right\}$, with $f_{1}, f_{2}: Y \rightarrow \mathbb{R}$ with the cardinality of $Y$ finite.

$$
\begin{aligned}
(5) & =\sum_{e \in E_{\mathbf{n}_{0}}}\left(\lambda^{e}\left(\zeta_{1}^{0}\right)-\lambda^{e}\left(\zeta_{2}^{0}\right)\right)\left(\min _{u \in U\left(\mathbf{n}_{0}, e\right)} \widehat{v}_{\mathbf{n}_{0}+u}\left(s_{0}, \zeta_{2}^{0} \circ u\right)-\widehat{v}_{\mathbf{n}_{0}}\left(s_{0}, \zeta_{2}^{0}\right)\right) \\
& \leq Q \sum_{e \in E_{\mathbf{n}_{0}}}\left(\lambda^{e}\left(\zeta_{1}^{0}\right)-\lambda^{e}\left(\zeta_{2}^{0}\right)\right)
\end{aligned}
$$

where $Q \equiv \max _{e \in E_{\mathbf{n}_{0}}}\left\{\min _{u \in U\left(\mathbf{n}_{0}, e\right)} \widehat{v}_{\mathbf{n}_{0}+u}\left(s_{0}, \zeta_{2}^{0} \circ u\right)-\widehat{v}_{\mathbf{n}_{0}}\left(s_{0}, \zeta_{2}^{0}\right)\right\}<\infty$, because the value function is bounded by the boundedness property of the loss function $g$ (see (2.12) and (2.13)).

Remark: In the inequality analysis above we applied that $U\left(\mathbf{n}, \zeta_{1}, e\right)=U\left(\mathbf{n}, \zeta_{2}, e\right)$ denoted $U(\mathbf{n}, e)$, for all $\left(\mathbf{n}, \zeta_{1}, e\right)$ and $\left(\mathbf{n}, \zeta_{2}, e\right)$, (see $\left.(2.5)\right)$.

(Step 3) By taking the positive parameters, $\alpha$ and $\beta$, to go to zero, we get a contradiction.

Hence from $(4.41),(4.43)-(4.47)$, for any $0<\alpha, \beta<\epsilon_{0}$, we obtain that

$$
\begin{aligned}
0 & <2 \gamma \leq(1)+(2)+(4)+(5) \\
& \leq L\left(\sqrt{\alpha \eta^{\star}}+\sqrt{\beta \eta^{\star}}\right)+Q\left(\sum_{e \in E_{\mathbf{n}_{0}}}\left(\lambda^{e}\left(\zeta_{1}^{0}\right)-\lambda^{e}\left(\zeta_{2}^{0}\right)\right)\right) \\
& \leq L\left(\sqrt{\alpha \eta^{\star}}+\sqrt{\beta \eta^{\star}}\right)+L Q\left(\left|\mathbb{V}^{\triangle}\right|+\sum_{i=1}^{R} n_{0}^{i}\right) \| \zeta_{1}^{0}-\zeta_{2}^{0}||, \quad \text { by assumption (S4) }
\end{aligned}
$$

$$
\rightarrow 0, \quad \text { with } \alpha, \beta \rightarrow 0,
$$

which is a contradiction. Hence we proved the symmetric property (4.2), i.e. $v \leq \widehat{v}$. Then by the symmetric property of $v$ and $\widehat{v}$, we obtain that $v=\widehat{v}$. 
Corollary 4.1. Under assumptions (S1)-(S5), the value function (2.13) is the unique viscosity solution of the hybrid HJB equations (2.17).

The proof of Corollary 4.1 is straightforward by Proposition 3.1 and Theorem 4.1.

5. Conclusion. In this paper, we have studied the viscosity solutions for a class of hybrid HJB equations, developed in the stochastic optimal control for the call admission and routing control problems in loss networks, which is a collection of coupled first order PDEs, linked by sets of integral coefficients. Under mild conditions for event rate and loss functions, the proof of uniqueness of the viscosity solutions is given. As a consequence, a class of loss network OSC problems are solved by implementing the viscosity solutions of the underlying HJB PDEs.

\section{REFERENCES}

[1] E. Altman and G. Koole, On submodular value functions and complex dynamic programming, Stochastic Models, 14(1998), pp. 1051-1072.

[2] M. G. Crandall and P. L. Lions, Viscosity solutions of Hamilton-Jacobi, Trans. Amer. Math. Soc., 277(1983), pp. 1-42.

[3] M. H. A. DAvis, Markov Models and Optimization. Chapman and Hall, London, 1993.

[4] W. H. Fleming and R. W. Rishel, Deterministic and Stochastic Optimal Control, SpringerVerlag, New York, 1975.

[5] W. H. Fleming and H. M. Soner, Controlled Markov Processes and Viscosity Solutions, Springer-Verlag, New York, 1993.

[6] H. HaJeK, Optimal control of two interacting service stations, IEEE Transactions on Automatic Control, 29(1984), pp. 491-499.

[7] B. Heyman, Optimal operating policies for $\mathrm{M} / \mathrm{G} / 1$ queuing systems. Operations Research, 16:362-382, 1968.

[8] M. Huang, P. E. Caines, and R. P. Malhamé, Degenerate stochastic control problems with exponential costs and weakly coupled dynamics: viscosity solutions and a maximum principle, SIAM J. Control and Optimization, 44:1(2005), pp. 367-387.

[9] S. Stidham JR. And R. Weber, A survey of markov decision models for control of networks of queues, Queueing Systems, 13(1993), pp. 291-314.

[10] F. P. Kelly, Loss networks, Annals of Applied Probability, 1:3(1991), pp. 319-378.

[11] S. Lenhart and N. Yamada, Perron's method for viscosity solutions associated with piecewisedeterministic processes, Funkcialaj Ekvacioj, 34(1991), pp. 173-186.

[12] Z. Ma, P. E. Caines, and R. P. Malhamé, Stochastic control of network systems I: NETCAD state space structure 83 dynamics, In: 45th IEEE Int. Conf. Decision and Control, pages 2577-2582, San Diego, CA, Dec. 2006.

[13] Z. Ma, P. E. Caines, And R. P. Malhamé, Stochastic control of network systems II: NETCAD optimal control $\&$ the HJB equation, In: 45th IEEE Int. Conf. Decision and Control, pages 3236-3241, San Diego, CA, Dec. 2006.

[14] Z. Ma, P. E. Caines, And R. P. Malhamé, Control of loss network systems: Call admission and routing control, Technical Report G-2007-53, GERAD - HEC Montreal, http://www.gerad.ca/fichiers/cahiers/G-2007-53.pdf, Jul. 2007.

[15] Z. Ma, P. E. Caines, And R. P. Malhamé, On the viscosity solutions of hybrid HJB equations arising in loss network system control, Technical Report G-2007-54, GERAD - HEC 
Montreal, http://www.gerad.ca/fichiers/cahiers/G-2007-54.pdf, Jul. 2007.

[16] Z. Ma, P. E. Caines, And R. P. Malhamé, Admission and routing control of loss networks: Hybrid dynamic programming equations, Submitted to IEEE Trans. Automatic Control, Apr. 2008. Under revision.

[17] S. P. Sethi and Q. Zhang, Hierarchical Decision Making in Stochastic Manufacturing Systems, Birkhauser Boston, Cambridge, 1994.

[18] M. XiAo AND T. BASAR, Optimal control of piecewise deterministic nonlinear systems with controlled transitions: Viscosity solutions, their existence and uniqueness, In: 38th IEEE Conference on Decision and Control, pages 4712-4717, Phoenix, AZ, Dec. 1999.

[19] J. YE, Generalized Bellman-Hamilton-Jacobi Equations for Piecewise Deterministic Markov Processes, volume 197 of System Modelling and Optimization, Lecture Notes in Control and Information Sciences, pages 539-550. Springer Berlin, Heidelberg, 1994.

[20] J. Yong And X. Y. Zhou, Stochastic Controls: Hamiltonian Systems and HJB Equations. Springer-Verlag, New York, 1999. 